\title{
Multiple Access for Mobile-UAV Enabled Networks: Joint Trajectory Design and Resource Allocation
}

\author{
Fangyu Cui, Yunlong Cai, Zhijin Qin, Minjian Zhao, and Geoffrey Ye Li
}

\begin{abstract}
In this article, we investigate joint trajectory design and resource allocation algorithms to maximize the minimum average rate among ground users for unmanned aerial vehicle (UAV) communication systems, where both the orthogonal multiple access (OMA) and non-orthogonal multiple access (NOMA) modes are considered. We first formulate the problems for UAV communications with the OMA and NOMA modes, respectively, which contain binary variables and highly coupled nonconvex objective functions and constraints. In order to handle the challenging problems, we transform the original problems into more tractable forms and then develop novel algorithms based on penalty dual-decomposition (PDD) technique to solve them. Simulation results show that the proposed algorithms outperform the benchmarks.
\end{abstract}

Index Terms-UAV communications, trajectory optimization, resource allocation, NOMA.

\section{INTRODUCTION}

Non-orthogonal multiple access (NOMA) can improve the capacity and support massive connectivity in the fifth generation (5G) and Internet of Things (IoT) networks [1]-[4]. Different from traditional orthogonal multiple access (OMA), NOMA $^{1}$ utilizes power domain in multiplexing in order to support multiple users sharing the same resource block. As a result, more users can be served with limited resource blocks and the network capacity is also improved but at the cost of extra interference and additional complexity at the receiver. The basic NOMA has been first proposed in [5]. Then, various NOMA related techniques have been investigated, including multiple antenna based NOMA [6]-[8], cooperative NOMA [9]-[11], power allocation in NOMA [12]-[14], physical layer security in NOMA [15], and spectral and energy efficiency in NOMA [16]-[18].

Unmanned aerial vehicle (UAV) communications are also attracting increasing interest recently due to flexible deployment, high mobility, and low cost [19], [20]. The UAV can be either used as an aerial user equipment (UE) [21]-[24] or a base station (BS) [25]-[37]. In [21], a three-dimensional (3D) cellular network is introduced, where both the BS and UE are

F. Cui, Y. Cai and M. Zhao are with the College of Information Science and Electronic Engineering, Zhejiang University, Hangzhou 310027, China (e-mail: cfy531@zju.edu.cn; ylcai@zju.edu.cn; mjzhao@zju.edu.cn).

Z. Qin is with the School of Electronic Engineering and Computer Science, Queen Mary University of London, London E1 4NS, U.K. (email: z.qin@qmul.ac.uk).

G. Y. Li is with the School of Electrical and Computer Engineering, Georgia Institute of Technology, Atlanta, USA (e-mail: liye@ece.gatech.edu).

${ }^{1}$ Power domain NOMA [5] is considered in this article.
UAVs. In [22]-[24], the UAVs are regarded as aerial users and connected to the ground BSs of cellular networks. In [22], the pathloss between the UAV UE and the ground BS is measured. In [23] and [24], the uplink of the cellular-connected UAV network is investigated and the interference due to the unique air-to-ground channel is managed. While in [25]-[30], the UAVs are considered as aerial quasi-static BSs or relays to support ground users in a given area. The deployment and altitudes of the UAVs are optimized to improve the performance of the network with a limit number of UAVs. Mobile-UAV networks, with the moving UAV BS instead of hovering at a fixed place, are also investigated. In [31], the UAV relay flies in a circular trajectory and the resource allocation is optimized to maximize the energy efficiency. Recently, the UAV trajectory is also considered in optimization to further improve the capacity [32], [33], physical security [34], energy efficiency [35], [36], and average age-of-information [37]. In [37], the authors jointly optimize the trajectory as well as energy and service time allocations to minimize the average age-of-information for IoT networks.

Usually, the users are assumed to be multiplexed in a time division multiple access (TDMA) mode when performing UAV trajectory optimization [32], [34], [36], [37]. In TDMA, a user either communicates in a basic time slot or not. Therefore, the scheduling variable must be binary. However, for the algorithms already proposed in the literature, the scheduling variables are usually non-binary for optimal performance. One way to address the issue is to divide the original time slots into more sub-slots and assign fractional resource accordingly [32], [34], [36]. Nevertheless, dividing a large time slot into too many small time slots may increase the burden of synchronization and cause other practical issues. Motivated by this, we investigate the algorithm that can directly converge to binary solutions in this paper. On the other hand, NOMA with proper power allocation may achieve higher transmission rate than OMA [5], which can be potentially used in UAV networks. There have been several studies that investigate the application of NOMA in UAV networks [38]-[40]. The work in [38] analyzes the outage performance of mobile-UAV network with a fixed circular trajectory. In [39], NOMA is employed in the network where the UAV works as the static BS and optimize the altitude. However, to the best of our knowledge, the trajectory optimization for NOMA based UAV network has not been investigated yet. The challenge of this issue comes from the varying successive interference cancellation (SIC) order in NOMA detection during the flight of the UAV. In this 
paper, we adopt NOMA in mobile-UAV networks and jointly optimize the UAV trajectory and power allocation to maximize the capacity of the NOMA communications.

In this work, we investigate joint trajectory design and resource allocation to maximize the minimum average rate among ground users for mobile-UAV communication systems, where both the OMA and NOMA modes are considered. The constraints of optimization problems include initial and final locations, UAV speed, and the scheduling variables. The optimization problems contain highly coupled nonconvex objective functions and are hard to handle. Moreover, the binary discrete constraints make the problems more challenging. For NOMA, SIC detection is used at the receiver [5]. Therefore, we should also take signal detection order into consideration, which may vary during the flight of the UAV and should be updated according to the trajectory in the optimization.

In order to address the above challenges, we first introduce some auxiliary variables and transform the original problems into more tractable forms. Specifically, we equivalently transform the binary variables into continuous ones with some equality constraints. In order to tackle the equality constraints, we incorporate the corresponding augmented Lagrangian (AL) terms into the objective functions. Then, with the aid of penalty dual-decomposition (PDD) technique, we develop double-loop iterative algorithms to solve the problems.

The main contributions of this paper are summarized as follows:

1) We present mobile-UAV enabled communication networks in the OMA and NOMA modes, respectively, and then formulate the corresponding joint trajectory design and resource allocation problems.

2) We transform the original problems to identical ones in the sense of optimal solutions and develop the PDD based algorithms to solve them. The proposed algorithms guarantee to converge to the local stationary solutions of the transformed problems, which are feasible for the original mixed-integer problems and meet the necessary conditions of optimal solutions.

3) We provide the complexity analysis of the algorithms and show that the proposed algorithms outperform other benchmarks through simulations.

The rest of this paper is organized as follows. Section II presents the system model of the mobile-UAV communication networks and formulates the problems. Sections III and IV propose the PDD based optimization algorithms for the OMA and NOMA modes, respectively. Section V provides the complexity analysis of the algorithms and Section VI presents the simulation results. Finally, the paper is concluded in Section VII.

Notations: Scalars are denoted by lower-case letters, vectors are denoted by boldface lower-case letters, and matrices are denoted by boldface upper-case letters. For a matrix $\mathbf{A}, \mathbf{A}^{H}$, and $\mathbf{A}^{-1}$ denote the conjugate transpose and pseudo-inverse, respectively. Moreover, $|x|,\|\mathbf{x}\|$, and $\|\mathbf{X}\|$ denote the absolute value of a scalar $x$, the Euclidean norm of a vector $\mathbf{x}$, and the Frobenius norm of a matrix $\mathbf{X}$, respectively. The notations $\Re\{$.$\} and \Im\{$.$\} represent the real and imaginary parts$ of a variable, respectively. For spaces, $\mathbb{R}^{m \times n}$ denotes the space

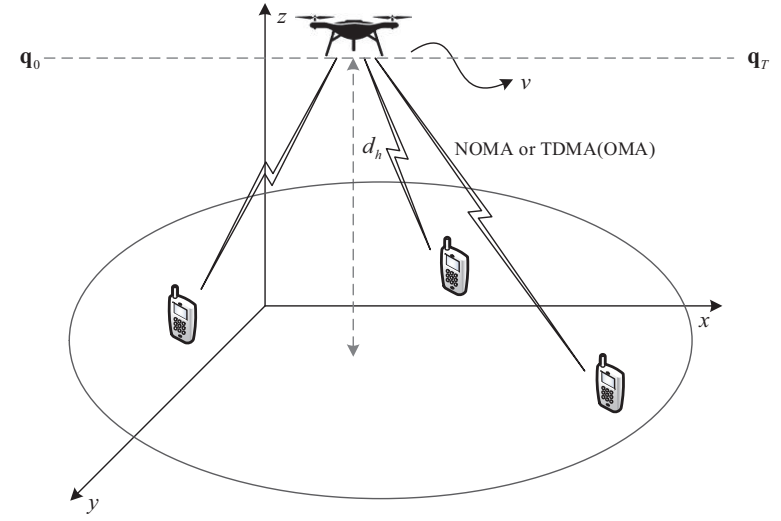

Fig. 1: The mobile-UAV communication systems accessed in either OMA mode or NOMA mode.

of $m \times n$ real matrices and $\mathbb{C}^{m \times n}$ correspondingly denotes the space of $m \times n$ complex matrices.

\section{SYSTEM MODEL AND PROBLEM FORMULATION}

In this section, we first introduce the models of the UAV communication systems with OMA and NOMA, respectively. Then, we formulate the optimization problems.

\section{A. System model}

Consider a downlink mobile-UAV communication system as shown in Fig. 1, where one UAV is employed to serve $K$ users on the ground. The UAV takes time $T$ to fly from the initial location $\mathbf{q}_{0}$ to the final location $\mathbf{q}_{T}$ at a fixed altitude $d_{h}$ above the ground ${ }^{2}$. The initial and final locations of the UAV are assumed to be fixed. Moreover, the maximum speed of the UAV is denoted as $V_{\max }$. All the users served by the UAV are assumed to share the same frequency band and multiplexed in other domains. The users are usually multiplexed in a TDMA mode, which can be regarded as a type of OMA scheme. Meanwhile, we can also employ NOMA in multiplexing to provide more access opportunities or higher transmission rates for each user. In the NOMA mode, all the users are served at the same time with different allocated power levels. At the receiver, SIC is employed to detect the signals for different users in ascending order of the channel gains.

We assume that the horizontal coordinate of user $k$ is denoted as $\mathbf{w}_{k} \in \mathbb{R}^{2}, k \in \mathcal{K} \triangleq\{1, \ldots, K\}$, and the time-varying horizontal coordinate of the UAV is denoted as $\mathbf{q}(t) \in \mathbb{R}^{2}, 0 \leq t \leq T$. To make the problem more tractable, the flight time $T$ is discretized into $N$ time slots and the horizontal trajectory coordinates of the UAV can be denoted as $\mathbf{q}(n) \in \mathbb{R}^{2}, n \in \mathcal{N} \triangleq\{1, \ldots, N\}$. Moreover, the trajectory should meet the following constraints determined by the maximum speed, initial and final locations, respectively:

$$
\begin{gathered}
\|\mathbf{q}(n+1)-\mathbf{q}(n)\| \leq V_{\max } \delta_{t}, n \in \mathcal{N}, \\
\mathbf{q}(1)=\mathbf{q}_{0},
\end{gathered}
$$

\footnotetext{
${ }^{2}$ Omnidirectional antenna and the dominating line-of-sight links are considered in this paper. The performance will increase when the UAV is closer to the ground users, i.e., flies at a lower altitude. Hence, the altitude of the $\mathrm{UAV}$ is set as the lowest allowed height.
} 
and

$$
\mathbf{q}(N)=\mathbf{q}_{T},
$$

where $\delta_{t}$ denotes the time slot length. The time slot length $\delta_{t}$ should be small enough so that the location of the UAV can be seen as approximately unchanged within each time slot. On the other hand, $\delta_{t}$ should not be too small with the consideration of complexity.

The distance between the UAV and user $k$ at time slot $n$ is denoted as

$$
d_{k}(n)=\sqrt{d_{h}^{2}+\left\|\mathbf{q}(n)-\mathbf{w}_{k}\right\|^{2}}, \forall n, k .
$$

Since there are no obstacles in the air, we can assume that the links between the UAV and the users are dominated by lineof-sight (LoS) links ${ }^{3}$ As a result, the channel gain follows the free-space model and path loss only depends on the distance. By presuming the path loss index to be 2, we can express the channel gain for user $k$ at time slot $n$ as

$$
h_{k}(n)=\sqrt{\frac{\rho_{0}}{d_{h}^{2}+\left\|\mathbf{q}(n)-\mathbf{w}_{k}\right\|^{2}}}, \forall n, k,
$$

where $\rho_{0}$ denotes the channel power gain at the reference distance $1 \mathrm{~m}$.

The main difference between the OMA and NOMA modes is the access scheme of ground users. In the OMA mode, the UAV only communicates with one user during each time slot so that all the transmission power is allocated to one user. Therefore, the signal-to-noise ratio (SNR) for user $k$ at time slot $n$ in OMA can be expressed as

$$
\gamma_{\mathrm{OMA}, k}(n) \triangleq \frac{\left|h_{k}(n)\right|^{2} P}{\sigma^{2}}=\frac{\rho_{0} P}{\sigma^{2}\left(d_{h}^{2}+\left\|\mathbf{q}(n)-\mathbf{w}_{k}\right\|^{2}\right)}, \forall n, k,
$$

where $\sigma^{2}$ denotes the variance of the additive white Gaussian noise (AWGN), and $P$ denotes the downlink transmission power of the UAV. While in the NOMA mode, the UAV serves multiple users at the same time. Moreover, the signals for users closer to the UAV and with higher channel gains are regarded as interference for those farther from the UAV and with lower channel gains due to the ascending order of the channel gains in SIC [5]. Therefore, we impose a type of binary indicator $\beta_{k, l}(n) \in\{0,1\}$ to indicate that the distance from the UAV to user $k$ is larger than that to user $l$ when $\beta_{k, l}(n)=1$, otherwise, $\beta_{k, l}(n)=0$. As a result, the signal-to-interference-plus-noise ratio (SINR) for user $k$ at time slot $n$ can be expressed as in (7), where $P_{k}(n)$ denotes the downlink transmission power allocated to user $k$ at time slot $n$ and should meet the following constraints

$$
P_{k}(n) \geq 0, \forall n, k,
$$

and

$$
\sum_{k \in \mathcal{K}} P_{k}(n) \leq P, \forall n
$$

\footnotetext{
${ }^{3}$ The problem will be more complicated if both LoS and non-LoS (NLoS) links are considered. For example, the study in [27] employs the probabilistic LoS/NLoS channel model, in which the altitude will affect the probabilities of having LoS connectivity for the ground users and should also be optimized. But, the problem framework does not change too much and our proposed algorithm can be directly extended to this case.
}

\section{B. OMA mode}

We formulate the optimization problem for the OMA mode here. The achievable average rate of user $k$ over all the $N$ time slots can be expressed as

$$
R_{\mathrm{OMA}, k} \triangleq \frac{1}{N} \sum_{n=1}^{N} \alpha_{k}(n) \log \left(1+\gamma_{\mathrm{OMA}, k}(n)\right),
$$

where $\alpha_{k}(n) \in\{0,1\}$ is imposed as the scheduling variable. When $\alpha_{k}(n)=1$, the time slot $n$ is allocated to user $k$. In order to achieve fairness, we maximize the achievable minimum average rate among the users by jointly optimizing the UAV trajectory, $\{\mathbf{q}(n)\}$, and user scheduling, $\left\{\alpha_{k}(n)\right\}$. Therefore, the problem can be formulated as

$$
\begin{aligned}
\max _{\left\{\mathbf{q}(n), \alpha_{k}(n)\right\}} & \min _{\forall k} R_{\mathrm{OMA}, k} \\
\text { s.t. } \quad & \|\mathbf{q}(n+1)-\mathbf{q}(n)\| \leq V_{\max } \delta_{t}, \forall n, \\
& \mathbf{q}(1)=\mathbf{q}_{0}, \mathbf{q}(N)=\mathbf{q}_{T}, \\
& \sum_{k \in \mathcal{K}} \alpha_{k}(n) \leq 1, \forall n, \\
& \alpha_{k}(n) \in\{0,1\}, \forall n, k,
\end{aligned}
$$

where (11d) and (11e) ensure that at most one user is scheduled at each time slot.

\section{NOMA mode}

We formulate the optimization problem for the NOMA mode here. The achievable average rate of user $k$ over all the $N$ time slots can be expressed as

$$
R_{\mathrm{NOMA}, k} \triangleq \frac{1}{N} \sum_{n=1}^{N} \log \left(1+\gamma_{\mathrm{NOMA}, k}(n)\right) .
$$

To maximize the achievable minimum average rate among the users, we jointly optimize the UAV trajectory, $\{\mathbf{q}(n)\}$, and power allocation, $\left\{P_{k}(n)\right\}$. This problem can be formulated as

$$
\begin{aligned}
\max _{\left\{\mathbf{q}(n), P_{k}(n)\right\}} & \min _{\forall k} R_{\mathrm{NOMA}, k} \\
\text { s.t. } . & \|\mathbf{q}(n+1)-\mathbf{q}(n)\| \leq V_{\max } \delta_{t}, \forall n, \\
& \mathbf{q}(1)=\mathbf{q}_{0}, \mathbf{q}(N)=\mathbf{q}_{T}, \\
& P_{k}(n) \geq 0, \forall n, k, \\
& \sum_{k \in \mathcal{K}} P_{k}(n) \leq P, \forall n, \\
& \beta_{k, l}(n)= \begin{cases}1, & \text { if } d_{k}(n)>d_{l}(n) \\
0, & \text { if } d_{k}(n)<d_{l}(n), \forall n, k, l \\
0 \text { or } 1, & \text { if } d_{k}(n)=d_{l}(n)\end{cases} \\
& \beta_{k, l}(n)+\beta_{l, k}(n)=1, \forall n, k, l,
\end{aligned}
$$

where (13f) ensures that the signals of stronger users are regarded as interference for signals of weaker users. When the channel gains are equal, either user can be chosen as the stronger one and the other is the weaker one. The constraint in $(13 \mathrm{~g})$ avoids the case where both users are regarded as stronger ones or weaker ones. 


$$
\gamma_{\mathrm{NOMA}, k}(n) \triangleq \frac{\left|h_{k}(n)\right|^{2} P_{k}(n)}{\sum_{l \in \mathcal{K}, l \neq k} \beta_{k, l}(n)\left|h_{k}(n)\right|^{2} P_{l}(n)+\sigma^{2}}=\frac{\rho_{0} P_{k}(n)}{\sum_{l \in \mathcal{K}, l \neq k} \rho_{0} \beta_{k, l}(n) P_{l}(n)+\sigma^{2}\left(d_{h}^{2}+\left\|\mathbf{q}(n)-\mathbf{w}_{k}\right\|^{2}\right)}, \forall n, k,
$$

Both problems in (11) and (13) contain binary variables and highly coupled nonconvex terms in the objective function, which makes it hard to find the global optimal solution. Moreover, the problem in (13) is even more challenging due to the additional binary indicators $\left\{\beta_{k, l}(n)\right\}$ determined by the trajectory variables. Therefore, we transform the problems into more tractable forms and propose PDD based joint optimization schemes to find the local stationary solutions in the following sections. The basic framework of the PDD method can be found in Appendix A. Note that if nonconvex constraints exist in the problem, concave-convex procedure (CCCP) method [41] can be employed in the inner loops of the PDD method to handle them. We summarize the procedures on developing the PDD-based algorithm in this paper in Fig. 2.

\section{OPTIMIZATION FOR OMA MODE}

In this section, we first transform the original problem for the OMA mode, i.e., (11), into an equivalent one that is more tractable. Then we develop a PDD based algorithm to solve it.

\section{A. Problem transformation}

First, in order to handle the highly coupled objective function, we introduce the auxiliary variables $\left\{\tilde{t}, t_{k}(n), \theta_{k}(n)\right.$, $\left.\tilde{\theta}_{k}(n)\right\}$ and transform the problem in (11) as

$$
\begin{array}{ll}
\max _{\mathcal{Z}_{\mathrm{OMA}}} & \tilde{t} \\
\text { s.t. } & \sum_{n=1}^{N} t_{k}(n) \geq \tilde{t}, \forall k, \\
& \alpha_{k}(n) \tilde{\theta}_{k}(n) \geq t_{k}(n), \forall n, k, \\
& \log \left(1+\theta_{k}(n)\right) \geq \tilde{\theta}_{k}(n), \forall n, k, \\
& \gamma_{\mathrm{OMA}, k}(n) \geq \theta_{k}(n), \forall n, k, \\
& (11 \mathrm{~b})-(11 \mathrm{e}),
\end{array}
$$

where $\mathcal{Z}_{\mathrm{OMA}} \triangleq\left\{\tilde{t}, t_{k}(n), \mathbf{q}(n), \alpha_{k}(n), \theta_{k}(n), \tilde{\theta}_{k}(n)\right\}$. The equivalence between (11) and (14) is demonstrated in Appendix B.

Since the expression of SNR, i.e., $\gamma_{\mathrm{OMA}, k}(n)$, is in fractional form, the constraint in $(14 \mathrm{e})$ is hard to handle. Therefore, we introduce the auxiliary variables $\left\{\pi_{k}(n)\right\}$ as the upper bound of the expression $\left.\sigma^{2}\left(d_{h}^{2}+\left\|\mathbf{q}(n)-\mathbf{w}_{k}\right\|^{2}\right)\right)$, and then equivalently transform (14e) as the following two constraints

$$
\pi_{k}(n) \theta_{k}(n) \leq \rho_{0} P, \forall n, k,
$$

and

$$
\left.\sigma^{2}\left(d_{h}^{2}+\left\|\mathbf{q}(n)-\mathbf{w}_{k}\right\|^{2}\right)\right) \leq \pi_{k}(n), \forall n, k .
$$

Moreover, the binary variables $\left\{\alpha_{k}(n)\right\}$ are also hard to handle. Therefore, we introduce the auxiliary variables $\left\{\tilde{\alpha}_{k}(n)\right\}$ and equivalently transform the binary constraints in (11e) as

$$
\alpha_{k}(n)\left(1-\tilde{\alpha}_{k}(n)\right)=0, \forall n, k,
$$

and

$$
\alpha_{k}(n)=\tilde{\alpha}_{k}(n), \forall n, k .
$$

We can easily find that the equality constraints hold only when $\alpha_{k}(n)=\tilde{\alpha}_{k}(n) \in\{0,1\}$. Therefore, the actual feasible set of the solution is not changed if we utilize these two equality constraints, which are easier to tackle in the PDD based algorithm, to replace the binary constraints in (11e).

From the above discussion, we can finally transform the problem in (11) to the following equivalent problem

$$
\begin{aligned}
\max _{\tilde{\mathcal{Z}}_{\text {OMA }}} & \tilde{t} \\
\text { s.t. } & (14 \mathrm{~b})-(14 \mathrm{~d}),(15),(16), \\
& (17),(18),(11 \mathrm{~b})-(11 \mathrm{~d}),
\end{aligned}
$$

where $\tilde{\mathcal{Z}}_{\mathrm{OMA}} \triangleq\left\{\tilde{t}, t_{k}(n), \mathbf{q}(n), \alpha_{k}(n), \theta_{k}(n), \tilde{\theta}_{k}(n), \pi_{k}(n)\right.$, $\left.\tilde{\alpha}_{k}(n)\right\}$. We can observe that the objective function $\tilde{t}$ is a scalar continuously differentiable function. All the variables in $\tilde{\mathcal{Z}}_{\text {OMA }}$ can be searched in the real number field, which is a closed convex set. Moreover, the functions in inequality constraints (11b), (11d), (14b) - (14d), (15), and (16) are all differentiable. While the functions in equality constraints (11c), (17) and (18) are continuously differentiable. Therefore, the problem in (19) satisfies the general framework for the PDD method, which is shown in (45) in Appendix A.

\section{B. Algorithm}

In this subsection, we utilize the PDD method to solve the problem in (19). First, in order to tackle the equality constraints, we incorporate the corresponding AL terms into the objective function. In each outer loop, the penalty parameter, $\varrho$, or the dual variable, $\lambda$, will be updated according to the constraint violation. Then, by applying linearization, we approximate the nonconvex constraints of the AL problem as convex ones and propose an algorithm to solve the problem in each inner loop. Finally, we present the overall PDD based double-loop iterative algorithm.

1) AL problem: In order to address the equality constraints, we incorporate the corresponding $\mathrm{AL}$ terms into the objective function and obtain the following AL problem

$$
\begin{aligned}
\max _{\tilde{\mathcal{Z}}_{\mathrm{OMA}}} & \tilde{t}-\frac{1}{2 \varrho} \sum_{n=1}^{N} \sum_{k=1}^{K}\left(\left|\alpha_{k}(n)-\tilde{\alpha}_{k}(n)+\varrho \lambda_{1, k}(n)\right|^{2}\right. \\
& \left.\quad+\left|\alpha_{k}(n)\left(1-\tilde{\alpha}_{k}(n)\right)+\varrho \lambda_{2, k}(n)\right|^{2}\right) \\
\text { s.t. } & (14 \mathrm{~b})-(14 \mathrm{~d}),(15),(16),(11 \mathrm{~b})-(11 \mathrm{~d}) \\
& 0 \leq \alpha_{k}(n) \leq 1, \forall n, k
\end{aligned}
$$




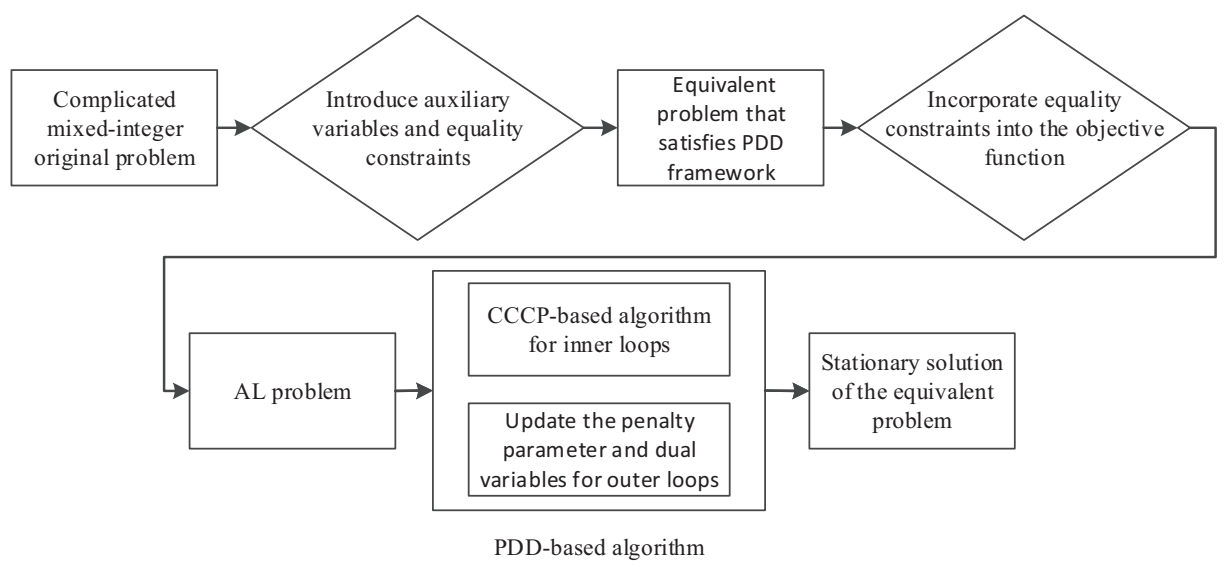

Fig. 2: The procedures on developing the PDD-based algorithm in this paper.

where (20c) has no influence on the optimality and is introduced to improve the convergence speed ${ }^{4}$. The PDD method utilizes a double-loop structure. In each outer loop, the dual variable, $\boldsymbol{\lambda}$, is updated when the constraint violation is under some threshold, otherwise the penalty coefficient, $\varrho$, is updated. While in the inner loops, we iteratively solve the AL problem in (20) to find the optimal solution of the current outer loop.

2) Proposed iterative algorithm for solving (20): As discussed above, in the outer loops we only need to update the dual variable, $\lambda$, or the penalty coefficient, $\varrho$. Therefore, the main procedure in the proposed algorithm is to solve the $\mathrm{AL}$ problem in (20) with the fixed $\boldsymbol{\lambda}$ and $\varrho$ in the inner loops. Referring to the PDD framework in Appendix A, the AL problem in (20) is solved in a block coordinate descent fashion in the inner loops. Since there exist nonconvex constraints in the AL problem, CCCP method is employed for the inner loops to approximate them as convex ones. In this case, the subproblems corresponding to the variable blocks are approximated as convex problems in each inner loop and we can easily provide the solution.

After the problem transformation in the previous subsection, we obtain the problem in (20) and can avoid the fractional form, binary variables, and the equality constraints. However, there are still some nonconvex constraints, such as (14c) and (15), that complicate the problem. By rewriting the nonconvex constraints into the difference-of-convex (DC) forms and then linearizing the subtracted convex terms, we can approximate them as convex constraints. For example, by applying first-order Taylor expansion in linearization, (15) can be transformed to the convex constraint in (21), where $\pi_{k}^{i}(n)$ and $\theta_{k}^{i}(n)$ denote the current values of the variables $\pi_{k}(n)$ and $\theta_{k}(n)$ in the $i$-th inner loop, respectively. The detailed derivation can be found in Appendix C. We can further rewrite the convex constraint in (21) as the second-order cone (SOC) form, which is shown in (22). The derivation of (22) is presented in Appendix D. Similarly, we can use the same

\footnotetext{
${ }^{4}$ The original searching range of $\alpha_{k}(n)$ is the whole real number field Since $\alpha_{k}(n)$ will finally converge to the binary solution, we can reduce the searching range to $[0,1]$ without affecting the optimality, which can significantly accelerate the convergence.
}

method to approximate (14c) to the SOC constraint in (23) Besides, following the same procedure in Appendix D, the convex constraint in (16) can also be rewritten as the following SOC form

$$
\left\|\left[\left(\mathbf{q}(n)-\mathbf{w}_{k}\right)^{T}, \frac{\pi_{k}(n)}{2 \sigma^{2}}-\frac{d_{h}^{2}}{2}-\frac{1}{2}\right]\right\| \leq \frac{\pi_{k}(n)}{2 \sigma^{2}}-\frac{d_{h}^{2}}{2}+\frac{1}{2} .
$$

From all the discussion above, the AL problem in (20) can be approximated to

$$
\begin{array}{cc}
\max _{\tilde{\mathcal{Z}}_{\mathrm{OMA}}} & \tilde{t}-\frac{1}{2 \varrho} \sum_{n=1}^{N} \sum_{k=1}^{K}\left(\left|\alpha_{k}(n)-\tilde{\alpha}_{k}(n)+\varrho \lambda_{1, k}(n)\right|^{2}\right. \\
& \left.+\left|\alpha_{k}(n)\left(1-\tilde{\alpha}_{k}(n)\right)+\varrho \lambda_{2, k}(n)\right|^{2}\right) \\
\text { s.t. } & (14 \mathrm{~b}),(14 \mathrm{~d}),(22)-(24) \\
& (11 \mathrm{~b})-(11 \mathrm{~d}),(20 \mathrm{c}), \forall n, k
\end{array}
$$

in the $i$-th inner loop according to the CCCP concept.

To solve the AL problem in (25), for each inner loop, we divide the variables $\tilde{\mathcal{Z}}_{\mathrm{OMA}}$ into two blocks and update each block successively. The first block contains variables $\left\{\tilde{\alpha}_{k}(n)\right\}$, which only exist in the objective function. Therefore, we can easily obtain optimal $\left\{\tilde{\alpha}_{k}(n)\right\}$ by closed-form solutions. We can use the convex programming toolbox CVX [42] to find the solution for the rest variables in the second block. Hence, the $i$-th inner loop of the proposed algorithm can be expressed as the following two steps:

Step 1: By fixing the variables in the second block, we can obtain the closed-form solutions for $\left\{\tilde{\alpha}_{k}(n)\right\}$ expressed as

$\tilde{\alpha}_{k}(n)=\frac{\alpha_{k}(n)+\alpha_{k}^{2}(n)+\varrho \lambda_{1, k}(n)+\varrho \lambda_{2, k}(n) \alpha_{k}(n)}{1+\alpha_{k}^{2}(n)}, \forall n, k$.

Step 2: In order to update the variables in the second block $\overline{\overline{\mathcal{Z}}_{\mathrm{OMA}}} \triangleq \tilde{\mathcal{Z}}_{\mathrm{OMA}} \backslash\left\{\tilde{\alpha}_{k}(n)\right\}$, we fix $\left\{\tilde{\alpha}_{k}(n)\right\}$ and utilize 


$$
\begin{gathered}
\frac{\left(\pi_{k}(n)+\theta_{k}(n)\right)^{2}}{4}-\rho_{0} P-\left(\frac{\left(\pi_{k}^{i}(n)-\theta_{k}^{i}(n)\right)\left(\pi_{k}(n)-\theta_{k}(n)\right)}{2}-\frac{\left(\pi_{k}^{i}(n)-\theta_{k}^{i}(n)\right)^{2}}{4}\right) \leq 0 \\
\left\|\left[\frac{\left(\pi_{k}(n)+\theta_{k}(n)\right)}{2}, \frac{\left(\pi_{k}^{i}(n)-\theta_{k}^{i}(n)\right)\left(\pi_{k}(n)-\theta_{k}(n)\right)}{4}-\frac{\left(\pi_{k}^{i}(n)-\theta_{k}^{i}(n)\right)^{2}}{8}+\frac{\rho_{0} P}{2}-\frac{1}{2}\right]\right\| \\
\leq \frac{\left(\pi_{k}^{i}(n)-\theta_{k}^{i}(n)\right)\left(\pi_{k}(n)-\theta_{k}(n)\right)}{4}-\frac{\left(\pi_{k}^{i}(n)-\theta_{k}^{i}(n)\right)^{2}}{8}+\frac{\rho_{0} P}{2}+\frac{1}{2} . \\
\left\|\left[\frac{\left(\alpha_{k}(n)-\tilde{\theta}_{k}(n)\right)}{2}, \frac{\left(\alpha_{k}^{i}(n)+\tilde{\theta}_{k}^{i}(n)\right)\left(\alpha_{k}(n)+\tilde{\theta}_{k}(n)\right)}{4}-\frac{\left(\alpha_{k}^{i}(n)+\tilde{\theta}_{k}^{i}(n)\right)^{2}}{8}-\frac{t_{k}(n)}{2}-\frac{1}{2}\right]\right\| \\
\leq \frac{\left(\alpha_{k}^{i}(n)+\tilde{\theta}_{k}^{i}(n)\right)\left(\alpha_{k}(n)+\tilde{\theta}_{k}(n)\right)}{4}-\frac{\left(\alpha_{k}^{i}(n)+\tilde{\theta}_{k}^{i}(n)\right)^{2}}{8}-\frac{t_{k}(n)}{2}+\frac{1}{2} .
\end{gathered}
$$

CVX to solve the following problem

$$
\begin{aligned}
\max _{\overline{\mathcal{Z}}_{\mathrm{OMA}}} & \tilde{t}-\frac{1}{2 \varrho} \sum_{n=1}^{N} \sum_{k=1}^{K}\left(\left|\alpha_{k}(n)-\tilde{\alpha}_{k}(n)+\varrho \lambda_{1, k}(n)\right|^{2}\right. \\
& \left.+\left|\alpha_{k}(n)\left(1-\tilde{\alpha}_{k}(n)\right)+\varrho \lambda_{2, k}(n)\right|^{2}\right) \\
\text { s.t. } & (14 \mathrm{~b}),(14 \mathrm{~d}),(22)-(24) \\
& (11 \mathrm{~b})-(11 \mathrm{~d}),(20 \mathrm{c}), \forall n, k
\end{aligned}
$$

The proposed iterative algorithm to solve the AL problem in (20), i.e., the algorithm for each inner loop of the PDD method is summarized in Algorithm 1 , where $\mathcal{L}_{\mathrm{OMA}, i}\left(\tilde{\mathcal{Z}}_{\mathrm{OMA}}\right)$ denotes the objective function of the AL problem in (20) in the $i$-th inner loop. Moreover, the overall PDD based algorithm is summarized in Algorithm 2, where goMA,r $\left(\tilde{\mathcal{Z}}_{\mathrm{OMA}}\right)$ denotes the vector that combines all functions in the equality constraints of the problem in (19) and $\eta_{r}$ denotes the constraint violation parameter. From the discussion in Appendix A, we can see that the proposed algorithm converges to a stationary solution of the problem in $(19)^{5}$. It is worth noting that there is no approximation or relaxation during the transformation from the original problem in (11) to the problem in (19). Hence, they share the same solutions. Moreover, since the obtained stationary solution meets the necessary conditions for the optimal solution of the problem in (19), it also meets the necessary conditions for the optimal solution of the original problem in (11).

\section{OPTIMIZATION FOR NOMA MODE}

In this section, we first transform the original problem for the NOMA mode, i.e., (13), into an equivalent and more tractable one. Then we develop a PDD based algorithm to solve it.

\footnotetext{
${ }^{5}$ In the absence of better alternative, it is readily seen that this proposed algorithm is the best choice for solving this optimization problem under study. The problem is NP-hard and it seems not possible to obtain the optimal solution based on the current optimization techniques. At the present time, the best we can do is to ensure the convergence to a stationary point for this kind of constrained optimization problem. The characterization of the performance gap between the optimal and iterative solutions remains an open problem for the future research.
}

\begin{tabular}{l}
\hline Algorithm 1 Proposed iterative algorithm for problem $(20)$ \\
\hline 1. Define the tolerance of accuracy $\delta$ and the maximum \\
number of iterations $N_{\max }$. Initialize the algorithm with \\
a feasible point $\tilde{\mathcal{Z}}_{\mathrm{OMA}}$. Set the iteration number $i=0$. \\
2. Repeat \\
- Update $\left\{\tilde{\alpha}_{k}(n)\right\}$ based on $(26)$. \\
- Update the variables in the second block based on \\
$\quad$ solving the problem in $(27)$. \\
- Update the iteration number $: i=i+1$. \\
3. Until $\frac{\left|\mathcal{L}_{\mathrm{OMA}, i+1}\left(\tilde{\mathcal{Z}}_{\mathrm{OMA}}\right)-\mathcal{L}_{\mathrm{OMA}, i}\left(\tilde{\mathcal{Z}}_{\mathrm{OMA}}\right)\right|}{\left|\mathcal{L}_{\mathrm{OMA}, i}\left(\tilde{\mathcal{Z}}_{\mathrm{OMA}}\right)\right|} \leq$, or the maximum \\
number of iterations is reached, i.e., $i>N_{\text {max }}$.
\end{tabular}

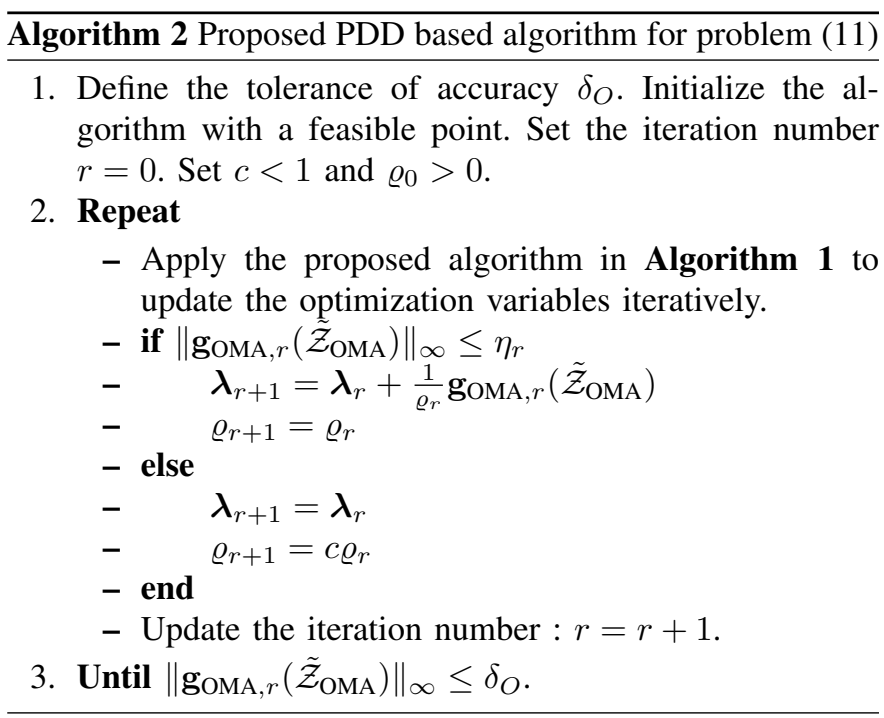

\section{A. Problem transformation}

Similar to Section III, we first handle the highly coupled objective function by introducing the auxiliary variables 
$\left\{\tilde{t}, t_{k}(n), \theta_{k}(n)\right\}$ and transforming the problem in (13) as

$$
\begin{aligned}
\max _{\mathcal{Z}_{\mathrm{NOMA}}} & \tilde{t} \\
\text { s.t. } & \sum_{n=1}^{N} t_{k}(n) \geq \tilde{t}, \forall k, \\
& \log \left(1+\theta_{k}(n)\right) \geq t_{k}(n), \forall n, k, \\
& \gamma_{\mathrm{NOMA}, k}(n) \geq \theta_{k}(n), \forall n, k, \\
& (13 \mathrm{~b})-(13 \mathrm{~g}),
\end{aligned}
$$

where $\mathcal{Z}_{\mathrm{NOMA}} \triangleq\left\{\tilde{t}, t_{k}(n), \mathbf{q}(n), P_{k}(n), \theta_{k}(n)\right\}$. The proof of equivalence between (13) and (28) is same as that between (11) and (14) for OMA problem and can be referred in Appendix B.

Similar to the OMA case, we introduce the auxiliary variables $\left\{\pi_{k}(n)\right\}$ as the upper bound of the expression $\left.\sum_{l \in \mathcal{K}, l \neq k} \rho_{0} \beta_{k, l}(n) P_{l}(n)+\sigma^{2}\left(d_{h}^{2}+\left\|\mathbf{q}(n)-\mathbf{w}_{k}\right\|^{2}\right)\right)$, and then equivalently transform (28d) as the following two constraints

$$
\pi_{k}(n) \theta_{k}(n) \leq \rho_{0} P_{k}(n), \forall n, k,
$$

and

$$
\begin{aligned}
\sum_{l \in \mathcal{K}, l \neq k} \rho_{0} \beta_{k, l}(n) P_{l}(n)+\sigma^{2}\left(d_{h}^{2}+\right. & \left.\left.\left\|\mathbf{q}(n)-\mathbf{w}_{k}\right\|^{2}\right)\right) \\
& \leq \pi_{k}(n), \forall n, k .
\end{aligned}
$$

To deal with the complicated constraint in (13f), we regard $\left\{\beta_{k, l}(n)\right\}$ as auxiliary variables. Then, by introducing the auxiliary variables $\left\{\tilde{\pi}_{k}(n)\right\}$ as the upper bound of $d_{h}^{2}+$ $\left\|\mathbf{q}(n)-\mathbf{w}_{k}\right\|^{2}$, we can equivalently transform (13f) as the following constraints

$$
\begin{gathered}
\beta_{k, l}(n) \in\{0,1\}, \forall n, k, \\
d_{h}^{2}+\left\|\mathbf{q}(n)-\mathbf{w}_{k}\right\|^{2} \leq \tilde{\pi}_{k}(n), \forall n, k, l,
\end{gathered}
$$

and

$$
\beta_{k, l}(n) \tilde{\pi}_{l}(n) \leq d_{h}^{2}+\left\|\mathbf{q}(n)-\mathbf{w}_{k}\right\|^{2}, \forall n, k, l \neq k .
$$

The process of the transformation can be found in Appendix E. In order to handle the binary variables $\left\{\beta_{k, l}(n)\right\}$, we introduce the auxiliary variables $\left\{\tilde{\beta}_{k, l}(n)\right\}$ and equivalently transform (31) as the following constraints

$$
\beta_{k, l}(n)\left(1-\tilde{\beta}_{k, l}(n)\right)=0, \forall n, k, l,
$$

and

$$
\beta_{k, l}(n)=\tilde{\beta}_{k, l}(n), \forall n, k, l .
$$

From the discussion above, we can finally transform the problem in (13) to the following equivalent problem

$$
\begin{aligned}
\max _{\tilde{\mathcal{Z}}_{\text {NOMA }}} & \tilde{t} \\
\text { s.t. } & (28 \mathrm{~b}),(28 \mathrm{c}),(29),(30), \\
& (32)-(35),(13 \mathrm{~b})-(13 \mathrm{e}),(13 \mathrm{~g}),
\end{aligned}
$$

where $\tilde{\mathcal{Z}}_{\mathrm{NOMA}} \triangleq\left\{\tilde{t}, t_{k}(n), \mathbf{q}(n), P_{k}(n), \theta_{k}(n), \pi_{k}(n), \beta_{k, l}(n)\right.$, $\left.\tilde{\pi}_{k}(n), \tilde{\beta}_{k, l}(n)\right\}$. Similar to the problem in (19), the problem in (36) also satisfies the general framework for the PDD method, which is shown in (45) in Appendix A.

\section{B. Algorithm}

Similar to Section III-B, we first incorporate the corresponding AL terms into the objective function to tackle the equality constraints. Then, by applying linearization, we approximate the nonconvex constraints of the AL problem as convex ones and propose an algorithm to solve the problem in each inner loop. Finally, the overall PDD based double-loop iterative algorithm is summarized.

1) AL problem: In order to address the equality constraints, we incorporate the corresponding AL terms into the objective function and obtain the AL problem in (37), where (37c) has no influence on the optimality and is introduced to improve the convergence speed. In the inner loops of the PDD method, we iteratively solve the AL problem in (37) to find the optimal solution of the current outer loop.

2) Proposed iterative algorithm for solving (37): After the problem transformation in the previous subsection, we obtain the problem in (37) and avoid the fractional form, binary variables, and equality constraints. Moreover, by following the same procedure in Appendices $\mathrm{C}$ and $\mathrm{D}$, we can approximate the nonconvex constraints of the problem in (37) as convex ones and then convert them into the SOC forms. As a result, (29), (30), and (33) can be approximated as the SOC constraints in (38)-(40), respectively. Besides, according to Appendix D, the convex constraint in (32) can also be rewritten as the following SOC form

$$
\left\|\left[\left(\mathbf{q}(n)-\mathbf{w}_{k}\right)^{T}, \frac{\tilde{\pi}_{k}(n)}{2}-\frac{d_{h}^{2}}{2}-\frac{1}{2}\right]\right\| \leq \frac{\tilde{\pi}_{k}(n)}{2}-\frac{d_{h}^{2}}{2}+\frac{1}{2} .
$$

From the transformation above, the AL problem in (37) can be approximated to

$$
\begin{aligned}
\max _{\tilde{\mathcal{Z}}_{\mathrm{NOMA}}} & \tilde{t} \\
- & \frac{1}{2 \varrho} \sum_{n=1}^{N} \sum_{k=1}^{K}\left(\sum_{l=1}^{K}\left|\beta_{k, l}(n)-\tilde{\beta}_{k, l}(n)+\varrho \lambda_{1, k, l}(n)\right|^{2}\right. \\
& +\sum_{l=1}^{K}\left|\beta_{k, l}(n)\left(1-\tilde{\beta}_{k, l}(n)\right)+\varrho \lambda_{2, k, l}(n)\right|^{2} \\
& -\sum_{l=1}^{k-1}\left(\left|\beta_{k, l}(n)+\beta_{l, k}(n)-1+\varrho \lambda_{3, k, l}(n)\right|^{2}\right)
\end{aligned}
$$

s.t. $\quad(28 b),(28 c),(38)-(41)$,

$$
(13 \mathrm{~b})-(13 \mathrm{e}),(37 \mathrm{c}), \forall n, k, l \text {, }
$$

in the $i$-th inner loop.

To solve the AL problem in (42) for each inner loop, we divide variables $\tilde{\mathcal{Z}}_{\text {NOMA }}$ into two blocks and update each successively. The first block only contains variables $\left\{\tilde{\beta}_{k, l}(n)\right\}$ that can be easily optimized by closed-form solutions. We can utilize CVX to find the solution for the rest variables in the second block. Hence, the $i$-th inner loop of the proposed algorithm can be expressed as the following two steps: 


$$
\begin{aligned}
& \max _{\tilde{\mathcal{Z}}_{\mathrm{NOMA}}} \tilde{t}-\frac{1}{2 \varrho} \sum_{n=1}^{N} \sum_{k=1}^{K} \sum_{l=1}^{K}\left(\left|\beta_{k, l}(n)-\tilde{\beta}_{k, l}(n)+\varrho \lambda_{1, k, l}(n)\right|^{2}+\left|\beta_{k, l}(n)\left(1-\tilde{\beta}_{k, l}(n)\right)+\varrho \lambda_{2, k, l}(n)\right|^{2}\right) \\
& -\frac{1}{2 \varrho} \sum_{n=1}^{N} \sum_{k=1}^{K} \sum_{l=1}^{k-1}\left(\left|\beta_{k, l}(n)+\beta_{l, k}(n)-1+\varrho \lambda_{3, k, l}(n)\right|^{2}\right) \\
& \text { s.t. (28b), (28c), (29), (30), (32), (33), (13b) - (13e), } \\
& 0 \leq \beta_{k, l}(n) \leq 1, \forall n, k, l \text {, } \\
& \left\|\left[\frac{\left(\pi_{k}(n)+\theta_{k}(n)\right)}{2}, \frac{\left(\pi_{k}^{i}(n)-\theta_{k}^{i}(n)\right)\left(\pi_{k}(n)-\theta_{k}(n)\right)}{4}-\frac{\left(\pi_{k}^{i}(n)-\theta_{k}^{i}(n)\right)^{2}}{8}+\frac{\rho_{0} P_{k}(n)}{2}-\frac{1}{2}\right]\right\| \\
& \leq \frac{\left(\pi_{k}^{i}(n)-\theta_{k}^{i}(n)\right)\left(\pi_{k}(n)-\theta_{k}(n)\right)}{4}-\frac{\left(\pi_{k}^{i}(n)-\theta_{k}^{i}(n)\right)^{2}}{8}+\frac{\rho_{0} P_{k}(n)}{2}+\frac{1}{2} \text {. } \\
& \|\left[\sum_{l \in \mathcal{K}, l \neq k}\left(\frac{\left(\beta_{k, l}^{i}(n)-P_{l}^{i}(n)\right)\left(\beta_{k, l}(n)-P_{l}(n)\right)}{4}-\frac{\left(\beta_{k, l}^{i}(n)-P_{l}^{i}(n)\right)^{2}}{8}\right)+\frac{\pi_{k}(n)-\sigma^{2} d_{h}^{2}}{2 \rho_{0}}-\frac{1}{2}, \frac{\sigma}{\sqrt{\rho_{0}}}\left(\mathbf{q}(n)-\mathbf{w}_{k}\right)^{T},\right. \\
& \left.\frac{\left(\beta_{k, 1}(n)+P_{1}(n)\right)}{2}, \ldots, \frac{\left(\beta_{k, k-1}(n)+P_{k-1}(n)\right)}{2}, \frac{\left(\beta_{k, k+1}(n)+P_{k+1}(n)\right)}{2}, \ldots, \frac{\left(\beta_{k, K}(n)+P_{K}(n)\right)}{2}\right] \| \leq \frac{1}{2} \\
& +\frac{\pi_{k}(n)-\sigma^{2} d_{h}^{2}}{2 \rho_{0}}+\sum_{l \in \mathcal{K}, l \neq k}\left(\frac{\left(\beta_{k, l}^{i}(n)-P_{l}^{i}(n)\right)\left(\beta_{k, l}(n)-P_{l}(n)\right)}{4}-\frac{\left(\beta_{k, l}^{i}(n)-P_{l}^{i}(n)\right)^{2}}{8}\right), \\
& \|\left[\frac{\beta_{k, l}(n)+\tilde{\pi}_{l}(n)}{2}, \frac{\left(\beta_{k, l}^{i}(n)-\tilde{\pi}_{l}^{i}(n)\right)\left(\beta_{k, l}(n)-\tilde{\pi}_{l}(n)\right)}{4}-\frac{\left(\beta_{k, l}^{i}(n)-\tilde{\pi}_{l}^{i}(n)\right)^{2}}{8}+\left(\mathbf{q}^{i}(n)-\mathbf{w}_{k}\right)^{T}\left(\mathbf{q}(n)-\mathbf{q}^{i}(n)\right)\right. \\
& \left.+\frac{\left\|\mathbf{q}^{i}(n)-\mathbf{w}_{k}\right\|^{2}}{2}+\frac{d_{h}^{2}}{2}-\frac{1}{2}\right] \| \leq \frac{\left(\beta_{k, l}^{i}(n)-\tilde{\pi}_{l}^{i}(n)\right)\left(\beta_{k, l}(n)-\tilde{\pi}_{l}(n)\right)}{4}-\frac{\left(\beta_{k, l}^{i}(n)-\tilde{\pi}_{l}^{i}(n)\right)^{2}}{8} \\
& +\left(\mathbf{q}^{i}(n)-\mathbf{w}_{k}\right)^{T}\left(\mathbf{q}(n)-\mathbf{q}^{i}(n)\right)+\frac{\left\|\mathbf{q}^{i}(n)-\mathbf{w}_{k}\right\|^{2}}{2}+\frac{d_{h}^{2}}{2}+\frac{1}{2} .
\end{aligned}
$$

Step 1: By fixing the variables in the second block, we can obtain the closed-form solutions for $\left\{\tilde{\beta}_{k, l}(n)\right\}$ expressed as

$\tilde{\beta}_{k, l}(n)=\frac{\beta_{k, l}(n)+\beta_{k, l}^{2}(n)+\varrho \lambda_{1, k, l}(n)+\varrho \lambda_{2, k, l}(n) \beta_{k, l}(n)}{1+\beta_{k, l}^{2}(n)}$,

$\forall n, k, l$.

Step 2: In order to update the variables in the second block $\overline{\mathcal{Z}}_{\text {NOMA }} \triangleq \tilde{\mathcal{Z}}_{\text {NOMA }} \backslash\left\{\tilde{\beta}_{k, l}(n)\right\}$, we fix $\left\{\tilde{\beta}_{k, l}(n)\right\}$ and utilize CVX to solve the following problem

$$
\begin{aligned}
\max _{\overline{\mathcal{Z}}_{\mathrm{NOMA}}} & \tilde{t}-\frac{1}{2 \varrho} \sum_{n=1}^{N} \sum_{k=1}^{K}\left(\sum_{l=1}^{K}\left|\beta_{k, l}(n)-\tilde{\beta}_{k, l}(n)+\varrho \lambda_{1, k, l}(n)\right|^{2}\right. \\
& +\sum_{l=1}^{K}\left|\beta_{k, l}(n)\left(1-\tilde{\beta}_{k, l}(n)\right)+\varrho \lambda_{2, k, l}(n)\right|^{2} \\
& -\sum_{l=1}^{k-1}\left(\left|\beta_{k, l}(n)+\beta_{l, k}(n)-1+\varrho \lambda_{3, k, l}(n)\right|^{2}\right)
\end{aligned}
$$

s.t. $\quad(28 b),(28 c),(38)-(41)$,

(13b) - (13e), (37c), $\forall n, k, l$.

The proposed iterative algorithm to solve the AL problem in (37), i.e., the algorithm for each inner loop of the PDD method, can be referred in Algorithm 1. We only need to replace the set of variables $\tilde{\mathcal{Z}}_{\text {OMA }}$ by $\tilde{\mathcal{Z}}_{\text {NOMA }}$ and employ (43) and (44) in the iterations instead of (26) and (27). The overall PDD based algorithm for the NOMA mode is similar to that for the OMA mode, which can be referred in Algorithm 2. Besides applying the above proposed algorithm for inner iterations, we need to replace $\mathbf{g}_{\mathrm{OMA}, r}\left(\tilde{\mathcal{Z}}_{\mathrm{OMA}}\right)$ by $\mathbf{g}_{\mathrm{NOMA}, r}\left(\tilde{\mathcal{Z}}_{\mathrm{NOMA}}\right)$, which denotes the vector that combines all functions in the equality constraints of the problem in (36). From the discussion in Appendix A, the proposed algorithm converges to a stationary solution of the problem in (36). Since the problem in (36) and the original one in (13) share the same optimal solution, the obtained stationary solution also meets the necessary conditions for the optimal solution of the original problem shown in (13).

\section{Computational Complexity}

In this section, we analyze the complexity for the proposed PDD based algorithms. Here, we apply the basic elements of complexity analysis as in [43].

The complexity of the proposed PDD based algorithm for the OMA mode is dominated by solving the problem in (27), which contains $3 K N$ SOC constraints, including $K N$ SOC constraints of dimension 4 and $2 K N$ SOC constraints of dimension 3 . The number of variables $n_{1}$ is on the order 
of $O(K N+N)$. Therefore, the complexity is on the order of $I_{1} I_{2} O\left(n_{1} \sqrt{6 K N}\left(34 K N+n_{1}^{2}\right)\right)$, where $I_{1}$ and $I_{2}$ denote the numbers of the inner and the outer iterations, respectively.

The complexity of the proposed PDD based algorithm for the NOMA mode is dominated by solving the problem in (44), which contains $\left(K^{2}+2 K\right) N$ SOC constraints, including $K N$ SOC constraints of dimension $K+3, K N$ SOC constraints of dimension 4 , and $K^{2} N$ SOC constraints of dimension 3. The number of variables $n_{2}$ is on the order of $O\left(K^{2} N+K N+N\right)$. Therefore, the complexity of the algorithm for the NOMA mode is on the order of $I_{3} I_{4} O\left(n_{2} \sqrt{2\left(K^{2}+2 K\right) N}\left(\left(K^{3}+15 K^{2}+25 K\right) N+n_{2}^{2}\right)\right)$, where $I_{3}$ and $I_{4}$ denote the numbers of the inner and the outer iterations, respectively.

The complexities for the proposed algorithms are summarized in Table I. It can be seen that the complexity order of NOMA is larger than OMA mainly because the dimensions of variables $\left\{\beta_{k, l}(n)\right\}$ are $O\left(K^{2} N\right)$ while the dimensions of others are at most $O(K N)$.

\section{Simulation Results}

In this section, we utilize computer simulation to evaluate the performance of the proposed PDD based optimization algorithms for the OMA and NOMA modes. We consider a mobile-UAV communication system with $K=3$ users randomly and uniformly distributed within an area of $1 \times 1 \mathrm{~km}^{2}$. The UAV flies from the initial location, $\mathbf{q}_{0}=[0,500]^{T}$, to the final location, $\mathbf{q}_{T}=[0,-500]^{T}$, with the altitude $d_{h}=100 \mathrm{~m}$. Moreover, the maximum UAV speed and the time slot length are set as $V_{\max }=50 \mathrm{~m} / \mathrm{sec}$ and $\delta_{t}=0.5 \mathrm{sec}$, respectively. The channel gain is $\rho_{0}=-70 \mathrm{~dB}$ at the reference distance $1 \mathrm{~m}$ and the noise level is $\sigma^{2}=-110 \mathrm{dBm}$. The tolerance parameters are set as $\delta=\delta_{O}=10^{-6}$, the maximum number of the inner iterations, $N_{\max }=10$, the initial penalty parameter, $\varrho_{0}=10^{4}$, the decrease parameter, $c=0.3$, and the equality constraint violation tolerance parameter is set as $\eta_{r}=0.3^{r}$ in the $r$-th outer iteration.

First, we consider the convergence of the proposed algorithms for the OMA and NOMA modes. The flight time is assumed to be $T=25 \mathrm{sec}$ and the transmit power is set as $P=20 \mathrm{dBm}$. Fig. 3 shows the max-min rate versus the number of iterations. From the figure, both algorithms converge within 10 iterations. Fig. 4 shows the penalty value versus the number of iterations. From the figure, the penalty values of both algorithms decrease below $10^{-6}$ within 25 iterations. Therefore, the proposed PDD based optimization algorithms can effectively handle the complicated constraints in the OMA and NOMA problems, respectively.

In Fig. 5, we present the trajectories and speeds of the UAV optimized by the proposed algorithms for different modes and the corresponding flight time $T$. The transmit power is set as $P=20 \mathrm{dBm}$. Fig. 5a and Fig. $5 \mathrm{c}$ present the trajectories and speeds for the OMA and NOMA modes when $T=25 \mathrm{sec}$. From the figure, in the OMA mode, the UAV intends to fly close to each user successively for fairness. Moreover, the $\mathrm{UAV}$ always flies at the maximum speed, $V_{\max }$, to move close to each user since the flight time, $T$, is not large enough.

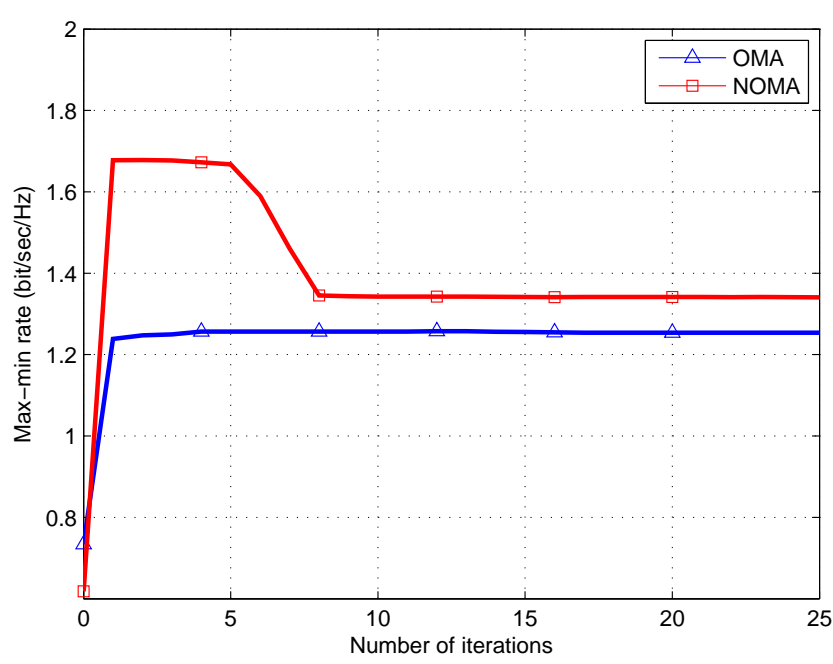

Fig. 3: The convergence performance of the proposed PDD based algorithms for the OMA and NOMA modes: max-min rate versus the number of iterations.

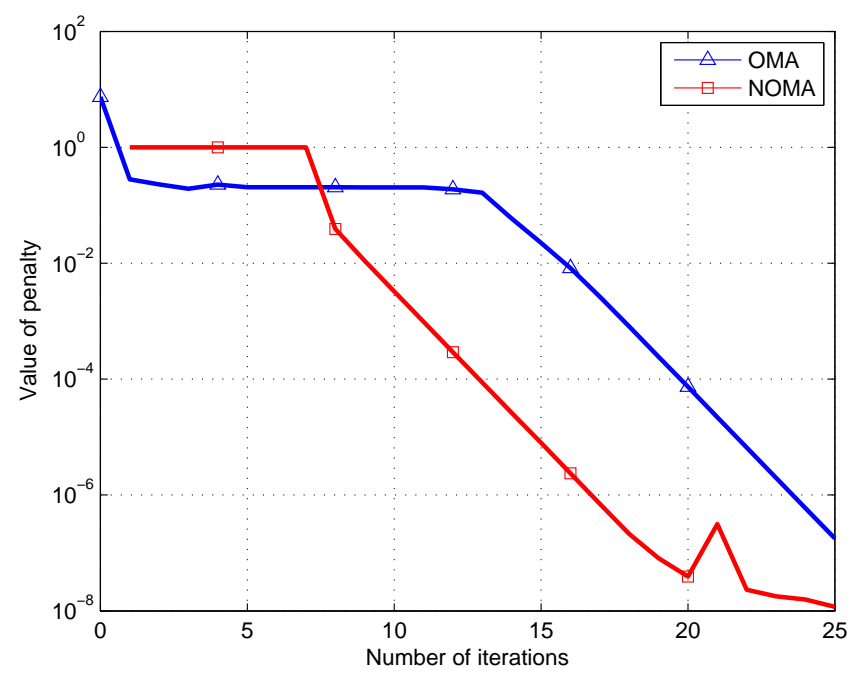

Fig. 4: The convergence performance of the proposed PDD based algorithms for the OMA and NOMA modes: penalty value versus the number of iterations.

However, in the NOMA mode, when $T$ is small so that the UAV cannot reach the remote users, more power is allocated to the remote users to increase the transmission rates to realize fairness. As a result, the UAV stays close to the nearby users for some time. Fig. 5b and Fig. 5d present the trajectories and speeds for the OMA and NOMA modes when $T=50 \mathrm{sec}$. Due to the longer flight time $T$, the UAV is able to reach the remote users. Therefore, the UAV flies over each user successively in both the OMA and NOMA modes. However, the trajectories and speeds are different because of different modes.

Fig. 6 illustrates the max-min rate performance versus flight time $T$ for different schemes, including the following algorithms for comparison: 
TABLE I: Complexity analysis for proposed algorithms

\begin{tabular}{|c|c|}
\hline Algorithms & Complexity Order \\
\hline OMA & $I_{1} I_{2} O\left(n_{1} \sqrt{6 K N}\left(34 K N+n_{1}^{2}\right)\right), \quad n_{1}=O(K N+N)$ \\
\hline NOMA & $I_{3} I_{4} O\left(n_{2} \sqrt{2\left(K^{2}+2 K\right) N}\left(\left(K^{3}+15 K^{2}+25 K\right) N+n_{2}^{2}\right)\right), \quad n_{2}=O\left(K^{2} N+K N+N\right)$ \\
\hline
\end{tabular}

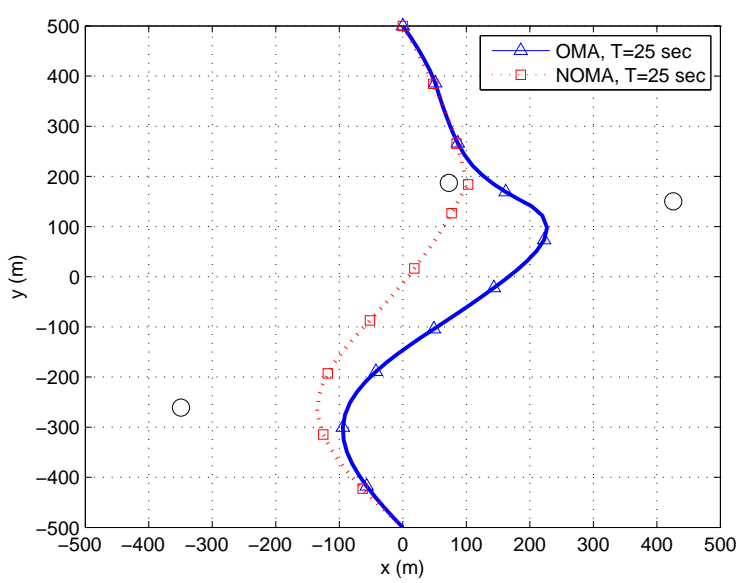

(a)

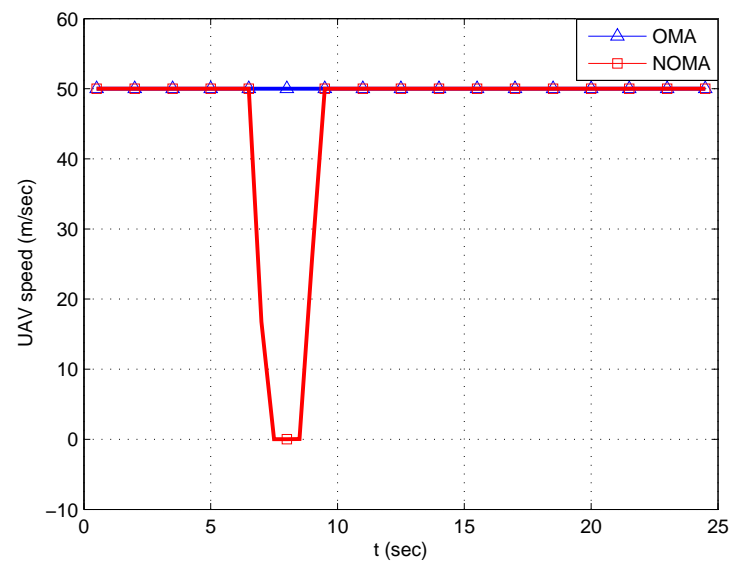

(c)

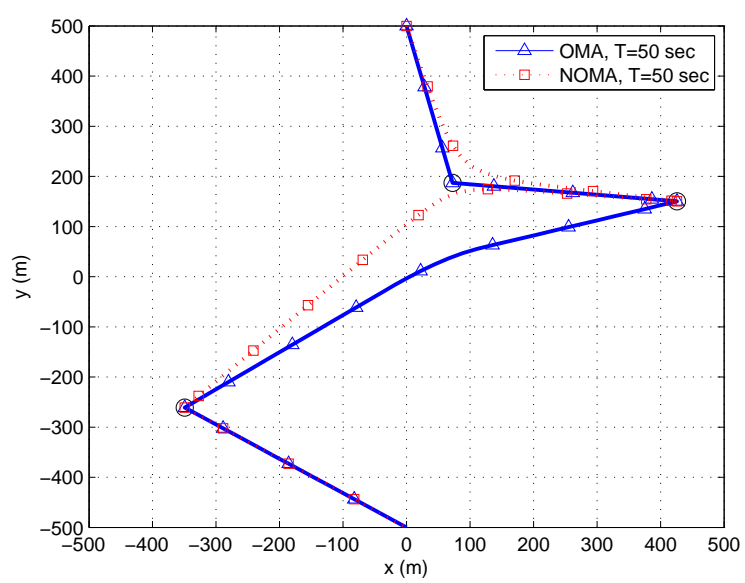

(b)

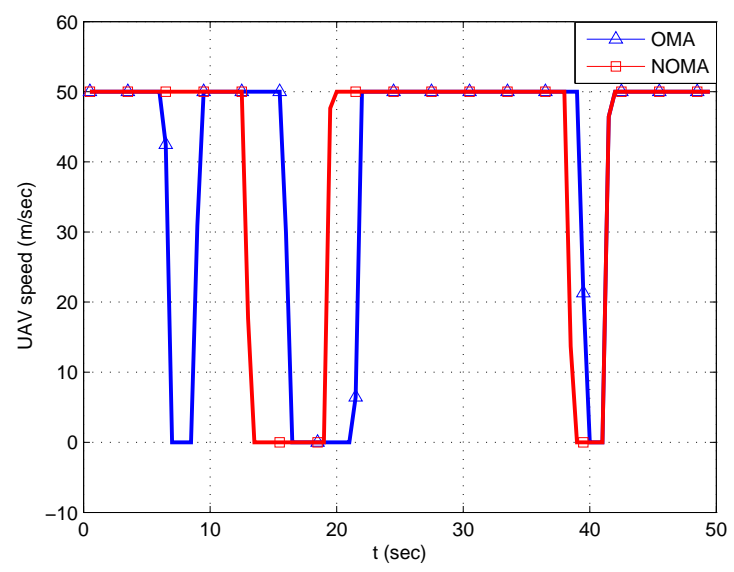

(d)

Fig. 5: Optimized UAV trajectories for: (a) $T=25 \mathrm{sec}$ and (b) $T=50 \mathrm{sec}$. (c) and (d) are the corresponding speeds of the UAV for scenarios in (a) and (b), respectively. Each trajectory is sampled every $2.5 \mathrm{sec}$ and the sampled points are marked with ' $\triangle$ ' and ' $\square$ ' for the OMA and NOMA modes, respectively. The user locations are marked by ' $\circ$ '.

- OMA traditional: the UAV flies in a straight line trajectory with a constant speed. As for scheduling, the users occupy the time slots one by one periodically.

- OMA straight traj: the UAV flies in a straight line trajectory with a constant speed while the user scheduling is optimized.

- OMA proposed: the trajectory and the user scheduling are jointly optimized.

- OMA upper bound: the flight time is assumed to be large enough so that the fraction of moving time between users is negligible. Therefore, the UAV can be regarded as hovering above each user sequentially and the upper bound of the performance is achieved.
- NOMA straight traj: the UAV flies in a straight line trajectory with a constant speed while the power allocation is optimized.

- NOMA proposed: the trajectory and the power allocation are jointly optimized.

"OMA proposed" and "NOMA proposed" are the algorithms proposed in this paper. While the others are benchmarks to show the effectiveness of our algorithms. The transmit power is set as $P=20 \mathrm{dBm}$. From the figure, the proposed joint optimization schemes achieve the best performance, followed by the schemes that only optimize resource allocation and the traditional OMA scheme without optimization. Moreover, the performance of joint optimization schemes improves with $T$ 


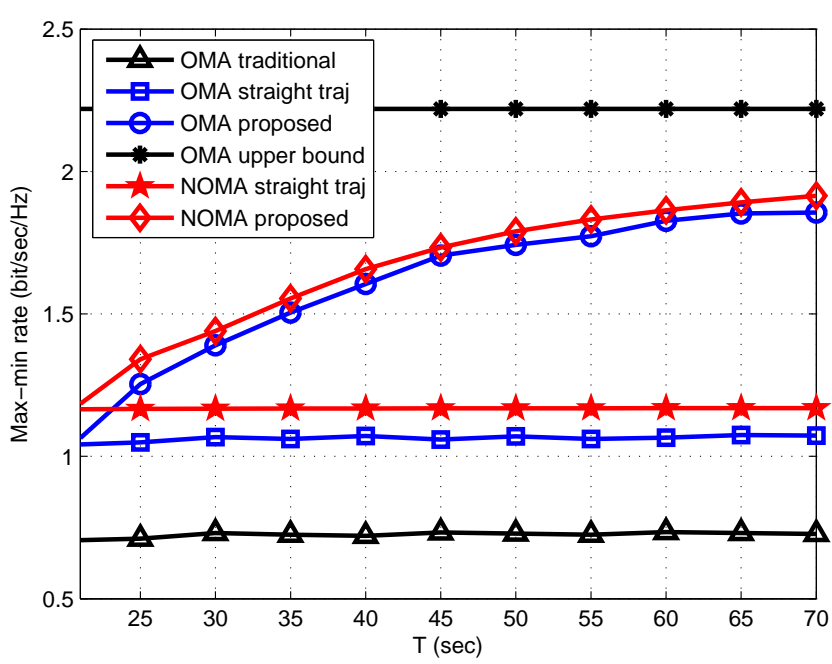

Fig. 6: Max-min rate performance versus flight time $T(P=$ $20 \mathrm{dBm})$.

since the UAV spends smaller fraction of time in moving from one user to another. When $T$ is large enough, the UAV can hover above each user sequentially so that the performance converges.

Fig. 7 illustrates the max-min rate performance versus the transmit power for different schemes. The flight time is set as $T=25 \mathrm{sec}$. From the figure, all schemes have improved performance with the transmit power. Among them, the proposed joint optimization schemes achieve the best performance, followed by the schemes that only optimize resource allocation and the traditional OMA scheme without optimization. Moreover, it is noted that the performance of the proposed NOMA scheme is better than the OMA mode. Besides, the gap between the NOMA mode and the OMA mode increases with the transmit power. When $P=30 \mathrm{dBm}$, the NOMA mode without trajectory optimization can achieve better performance than the OMA mode that employs joint optimization.

Fig. 8 shows the effectiveness of user scheduling optimization in our proposed algorithms by comparing them with the following benchmarks:

- OMA relaxed: the trajectory and the user scheduling are jointly optimized, where the binary scheduling variables $\left\{\alpha_{k}(n)\right\}$ are relaxed to continuous ones in $[0,1]$.

- NOMA relaxed: the trajectory and the power allocation are jointly optimized, where the binary variables $\left\{\beta_{k, l}(n)\right\}$ are relaxed to continuous ones in $[0,1]$.

- OMA random: the user scheduling is randomly generated while the trajectory is optimized.

- NOMA random: the SIC order is randomly generated while the trajectory and the power allocation are jointly optimized.

The flight time is set as $T=25 \mathrm{sec}$. From the figure, the performance of the proposed algorithms are very close to the relaxed benchmarks, which indicates that the bias caused by equalities does not significantly block the optimization. Moreover, the performance of "OMA random" and "NOMA

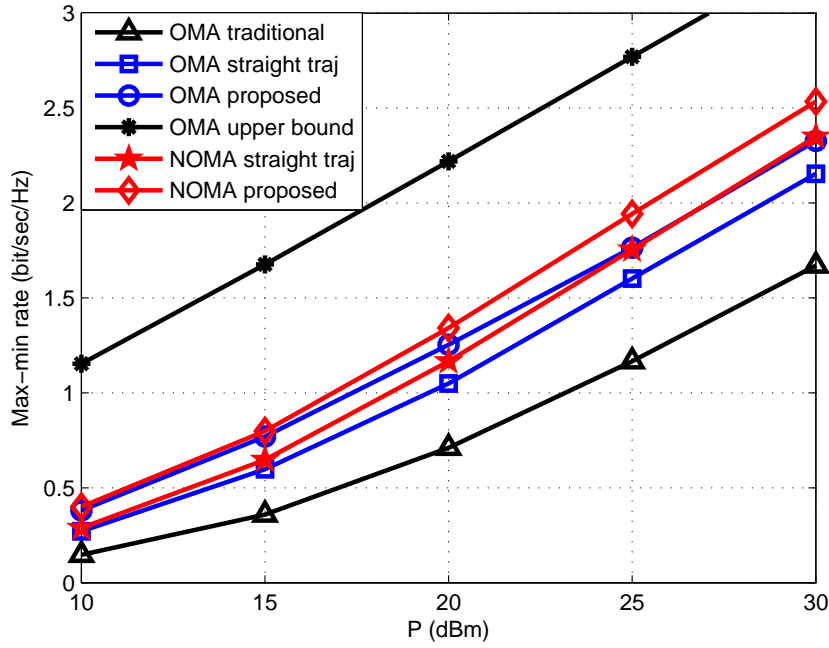

Fig. 7: Max-min rate performance versus transmit power $P(T=$ $25 \mathrm{sec})$.

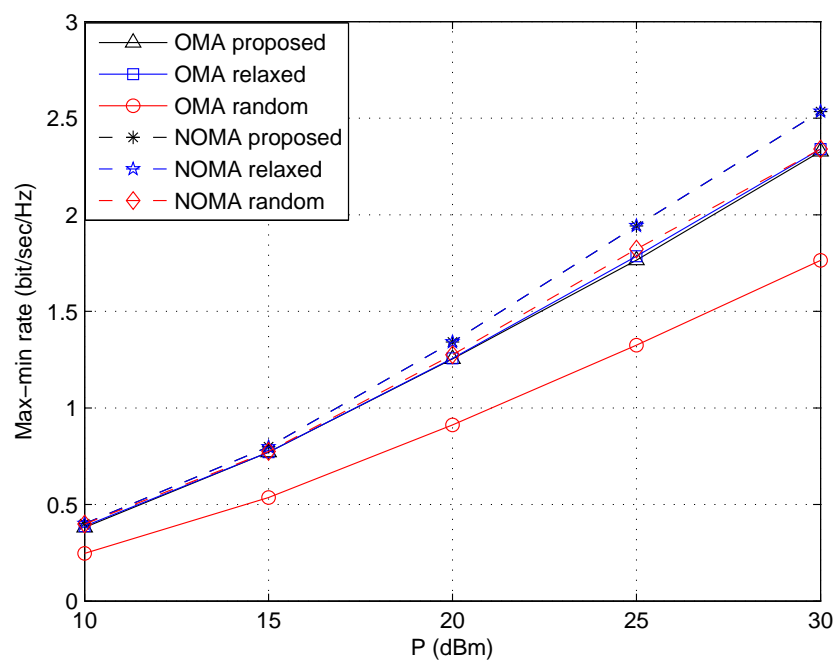

Fig. 8: Max-min rate performance versus transmit power $P(T=$ $25 \mathrm{sec})$.

random" is seriously degraded compared to our proposed algorithms and the relaxed benchmarks. Therefore, we can conclude that our proposed algorithms can well optimize the user scheduling.

\section{CONCLUSION}

In this paper, we have investigated the joint trajectory design and resource allocation for UAV-enabled multiple access systems. We have first formulated the problems to maximize the minimum average rate among multiple users for both the OMA and NOMA modes with the constraints of the UAV speed, initial and final locations, transmit power, and flight time. In order to handle the binary variables, highly coupled nonconvex terms in the objective functions and nonconvex constraints, we have introduced auxiliary variables and applied linearization to transform the original problems into more tractable forms 
with equality constraints. Then, we have proposed PDD based joint optimization algorithms to find local stationary solutions. We have also discussed the complexities of the proposed PDD based algorithms and used simulation results to show that the proposed algorithms outperform the benchmarks.

\section{APPENDIX A}

\section{PDD OPTIMIZATION FRAMEWORK}

Here, we briefly review PDD method in a general framework. Consider the following problem:

$$
\begin{array}{r}
(P) \quad \min _{\mathbf{x} \in \mathcal{X}} f(\mathbf{x}) \\
\text { s.t. } \mathbf{h}(\mathbf{x})=\mathbf{0}, \\
\mathbf{g}(\mathbf{x}) \leq \mathbf{0} .
\end{array}
$$

where $f(\mathbf{x})$ is a scalar continuously differentiable function and $\mathcal{X} \subseteq \mathbb{R}^{n}$ is a closed convex set. As for constraints, $\mathbf{h}(\mathbf{x}) \in \mathbb{R}^{p}$ is a vector of $p$ continuously differentiable functions, and $\mathbf{g}(\mathbf{x}) \in \mathbb{R}^{q}$ is a vector of $q$ differentiable while possibly nonconvex functions. In order to handle the equality constraints, we solve the following AL problem:

$$
\begin{aligned}
\left(P_{\varrho, \boldsymbol{\lambda}}\right) \quad \min _{\mathbf{x} \in \mathcal{X}} \mathcal{L}(\mathbf{x}) & \triangleq f(\mathbf{x})+\boldsymbol{\lambda}^{T} \mathbf{h}(\mathbf{x})+\frac{1}{2 \varrho}\|\mathbf{h}(\mathbf{x})\|^{2} \\
& =f(\mathbf{x})+\frac{1}{2 \varrho}\|\mathbf{h}(\mathbf{x})+\varrho \boldsymbol{\lambda}\|^{2} \\
\text { s.t. } \mathbf{g}(\mathbf{x}) & \leq \mathbf{0},
\end{aligned}
$$

where $\mathcal{L}(\mathbf{x})$ is the AL function with scalar penalty parameter $\varrho$ and dual variable $\lambda$. In particular, when $\varrho \rightarrow 0$, solving the above problem yields an identical solution to problem (45) [44]. The PDD method is a double-loop algorithm, where the inner loop solves the AL problem (46) via a block coordinate descent method while the outer loop updates the penalty parameter $\varrho$ or the dual variable $\lambda$ according to the constraint violation. The PDD framework is summarized in Algorithm 3. The detailed discussion about the convergence can be found in [45], [46]. It demonstrates that the sequence generated by the PDD method converges to a KKT (stationary) point of problem $(P)$.

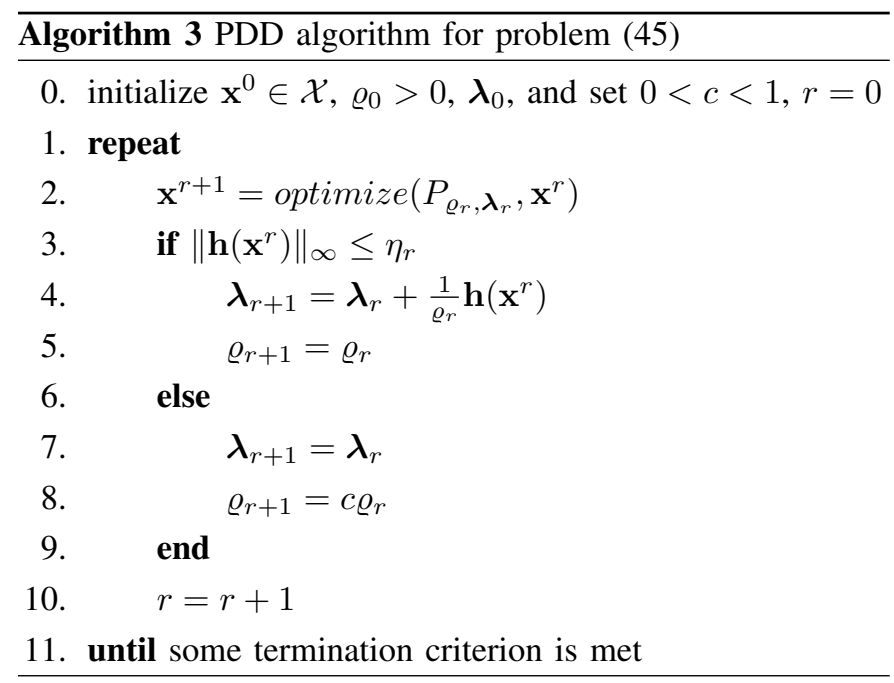

\section{APPENDIX B}

PROOF OF EQUIVALENCE BETWEEN (11) AND (14)

Let us first introduce variables $\left\{\tilde{\theta}_{k}(n)\right\}$ as the lower bound of $\log \left(1+\gamma_{\text {OMA }, k}(n)\right), \forall n, k$. Therefore, the problem (11) can be equivalently expressed as

$$
\begin{aligned}
\max _{\left\{\mathbf{q}(n), \alpha_{k}(n), \tilde{\theta}_{k}(n)\right\}} & \min _{\forall k} \sum_{n=1}^{N} \alpha_{k}(n) \tilde{\theta}_{k}(n) \\
\text { s.t. } \quad & \log \left(1+\gamma_{\mathrm{OMA}, k}(n)\right) \geq \tilde{\theta}_{k}(n), \\
& (11 \mathrm{~b})-(11 \mathrm{e}),
\end{aligned}
$$

Then, we introduce auxiliary variables $\theta_{k}(n)$ and $t_{k}(n)$ as the lower bounds of $\gamma_{\mathrm{OMA}, k}(n)$ and $\alpha_{k}(n) \tilde{\theta}_{k}(n)$, respectively. As a result, problem (47) can be further equivalently transformed to the following form

$$
\begin{array}{ll}
\max _{\mathcal{Z}} & \min _{\forall k} \sum_{n=1}^{N} t_{k}(n) \\
\text { s.t. } & \alpha_{k}(n) \tilde{\theta}_{k}(n) \geq t_{k}(n), \\
& \log \left(1+\theta_{k}(n)\right) \geq \tilde{\theta}_{k}(n), \\
& \gamma_{\mathrm{OMA}, k}(n) \geq \theta_{k}(n), \forall n, k, \\
& (11 \mathrm{~b})-(11 \mathrm{e}),
\end{array}
$$

where $\mathcal{Z}=\left\{\mathbf{q}(n), \alpha_{k}(n), \theta_{k}(n), \tilde{\theta}_{k}(n), t_{k}(n)\right\}$. Finally, by introducing variable $\tilde{t}$ as the lower bound of $\sum_{n=1}^{N} t_{k}(n), \forall k$, the problem (48) can be converted to problem (14). The proof of equivalence between (11) and (14) is completed.

\section{APPENDIX C \\ LINEARIZATION OF (15)}

Let us first transform (15) into the following DC form

$$
f_{1}(\mathbf{z})-f_{2}(\mathbf{z}) \leq 0,
$$

where

$$
\begin{gathered}
f_{1}(\mathbf{z})=\frac{\left(\pi_{k}(n)+\theta_{k}(n)\right)^{2}}{4}-\rho_{0} P, \\
f_{2}(\mathbf{z})=\frac{\left(\pi_{k}(n)-\theta_{k}(n)\right)^{2}}{4},
\end{gathered}
$$

and $\mathbf{z} \triangleq\left[\pi_{k}(n), \theta_{k}(n)\right]^{T}$. The convex function $f_{2}(\mathbf{z})$, which is subtracted, can be approximated to a linear function by the first-order Taylor expansion around the current point $\mathbf{z}^{i} \triangleq$ $\left[\pi_{k}^{i}(n), \theta_{k}^{i}(n)\right]^{T}$ in the $i$-th inner loop. As a result, we can obtain the approximated convex constraint as follows

$$
f_{1}(\mathbf{z})-\tilde{f}_{2}\left(\mathbf{z}^{i}, \mathbf{z}\right) \leq 0,
$$

where

$$
\begin{aligned}
\tilde{f}_{2}\left(\mathbf{z}^{i}, \mathbf{z}\right)= & f_{2}\left(\mathbf{z}^{i}\right)+2 \Re\left\{\nabla f_{2}^{H}\left(\mathbf{z}^{i}\right)\left(\mathbf{z}-\mathbf{z}^{i}\right)\right\} \\
= & \frac{\left(\pi_{k}^{i}(n)-\theta_{k}^{i}(n)\right)\left(\pi_{k}(n)-\theta_{k}(n)\right)}{2} \\
& -\frac{\left(\pi_{k}^{i}(n)-\theta_{k}^{i}(n)\right)^{2}}{4} .
\end{aligned}
$$

Substituting (50) and (53) into (52), we finally obtain the convex constraint (21). 
APPENDIX D

DERIVATION OF (22)

Let us first rewrite (21) as

$$
f_{3}(\mathbf{z}) \leq f_{4}(\mathbf{z}),
$$

where

$$
f_{3}(\mathbf{z})=\frac{\left(\pi_{k}(n)+\theta_{k}(n)\right)^{2}}{4}
$$

and

$$
\begin{array}{r}
f_{4}(\mathbf{z})=\frac{\left(\pi_{k}^{i}(n)-\theta_{k}^{i}(n)\right)\left(\pi_{k}(n)-\theta_{k}(n)\right)}{2} \\
-\frac{\left(\pi_{k}^{i}(n)-\theta_{k}^{i}(n)\right)^{2}}{4}+\rho_{0} P .
\end{array}
$$

It can be easily seen that $f_{3}(\mathbf{z})$ and $f_{4}(\mathbf{z})$ contain the quadratic terms and linear terms, respectively. We can equivalently rewrite (54) as

$$
f_{3}(\mathbf{z})+\frac{\left(f_{4}(\mathbf{z})-1\right)^{2}}{4} \leq \frac{\left(f_{4}(\mathbf{z})+1\right)^{2}}{4} .
$$

By taking the square root of both sides of (57), (21) can be converted to

$$
\left\|\left[\sqrt{f_{3}(\mathbf{z})}, \frac{f_{4}(\mathbf{z})-1}{2}\right]\right\| \leq \frac{f_{4}(\mathbf{z})+1}{2} .
$$

Substituting (55) and (56) into (58), we finally obtain the SOC constraint (22).

\section{APPENDIX E}

\section{TRANSFORMATION OF CONSTRAINT (13f)}

By regarding $\left\{\beta_{k, l}(n)\right\}$ as auxiliary variables, we rewrite (13f) as the following constraints

$$
\beta_{k, l}(n) \in\{0,1\}
$$

and

$\beta_{k, l}(n)\left(d_{h}^{2}+\left\|\mathbf{q}(n)-\mathbf{w}_{l}\right\|^{2}\right) \leq d_{h}^{2}+\left\|\mathbf{q}(n)-\mathbf{w}_{k}\right\|^{2}, \forall n, k, l \neq k$.

The first constraint (59) ensures that $\left\{\beta_{k, l}(n)\right\}$ are binary. Then, from (60) we can obtain $\beta_{k, l}(n)=0$ when $d_{h}^{2}+\| \mathbf{q}(n)-$ $\mathbf{w}_{l}\left\|^{2}>d_{h}^{2}+\right\| \mathbf{q}(n)-\mathbf{w}_{k} \|^{2}$, i.e., $d_{k}(n)<d_{l}(n)$. However, we cannot make sure that $\beta_{k, l}(n)=1$ when $d_{k}(n)>d_{l}(n)$. As a result, we take the constraint $(13 \mathrm{~g})$ into account to avoid the case where $\beta_{k, l}(n)=\beta_{l, k}(n)=0$. Moreover, when $d_{k}(n)=d_{l}(n)$, constraint (13g) also avoids the case where $\beta_{k, l}(n)=\beta_{l, k}(n)=1$.

Furthermore, the multiplication between the linear term and the quadratic term in constraint (60) is hard to handle. Therefore, by introducing auxiliary variables $\left\{\tilde{\pi}_{k}(n)\right\}$ as the upper bound of $d_{h}^{2}+\left\|\mathbf{q}(n)-\mathbf{w}_{k}\right\|^{2}$, (60) can be equivalently converted as

$$
d_{h}^{2}+\left\|\mathbf{q}(n)-\mathbf{w}_{k}\right\|^{2} \leq \tilde{\pi}_{k}(n),
$$

and

$$
\beta_{k, l}(n) \tilde{\pi}_{l}(n) \leq d_{h}^{2}+\left\|\mathbf{q}(n)-\mathbf{w}_{k}\right\|^{2}, \forall n, k, l \neq k .
$$

Finally, the constraint (13f) is transformed to constraints (31)(33).

\section{REFERENCES}

[1] Y. Cai, Z. Qin, F. Cui, G. Y. Li, and J. A. McCann, "Modulation and multiple access for 5G networks," IEEE Commun. Surv. Tut., vol. 20, no. 1, pp. 629-646, first quarter 2018.

[2] Y. Liu, Z. Qin, M. Elkashlan, Z. Ding, A. Nallanathan, and L. Hanzo, "Nonorthogonal multiple access for 5G and beyond," Proc. IEEE, vol. 105, no. 12, pp. 2347-2381, Dec. 2017.

[3] M. Shirvanimoghaddam, M. Dohler, and S. Johnson, "Massive nonorthogonal multiple access for cellular IoT: potentials and limitations," IEEE Commun. Mag., vol. 55, no. 9, pp. 55-61, Sep. 2017.

[4] Z. Qin, X. Yue, Y. Liu, Z. Ding, and A. Nallanathan, "User association and resource allocation in unified non-orthogonal multiple access enabled heterogeneous ultra dense networks," IEEE Commun. Mag., vol. 56, no. 6, pp. 86-92, June 2018.

[5] Y. Saito, Y. Kishiyama, A. Benjebbour, T. Nakamura, A. Li, and K. Higuchi, "Non-orthogonal multiple access (NOMA) for cellular future radio access," in Proc. IEEE Veh. Technol. Conf. (VTC Spring), Dresden, Germany, Jun. 2013, pp.1-5.

[6] Z. Ding, F. Adachi, and H. V. Poor, "The application of MIMO to nonorthogonal multiple access," IEEE Trans. Wireless Commun., vol. 15, no. 1, pp. 537-552, Jan. 2016.

[7] Z. Chen, Z. Ding, X. Dai, and G. K. Karagiannidis, "On the application of quasi-degradation to MISO-NOMA downlink," IEEE Trans. Signal Process., vol. 64, no. 23, pp. 6174-6189, Dec. 2016.

[8] Z. Ding, R. Schober, and H. V. Poor, "A general MIMO framework for NOMA downlink and uplink transmission based on signal alignment," IEEE Trans. Wireless Commun., vol. 15, no. 6, pp. 4438-4454, Jun. 2016.

[9] Z. Ding, M. Peng, and H. V. Poor, "Cooperative non-orthogonal multiple access in 5G systems," IEEE Commun. Lett., vol. 19, no. 8, pp. 1462 1465, Aug. 2015.

[10] Y. Tian, A. Nix, and M. Beach, "On the performance of opportunistic NOMA in downlink CoMP networks," IEEE Commun. Lett., vol. 20, no. 5, pp. 998-1001, May 2016.

[11] Z. Ding, H. Dai, and H. V. Poor, "Relay selection for cooperative NOMA,” IEEE Wireless Commun. Lett., vol. 5, no. 4, pp. 416-419, Aug. 2016.

[12] Z. Ding, Z. Yang, P. Fan, and H. V. Poor, "On the performance of nonorthogonal multiple access in 5G systems with randomly deployed users," IEEE Signal Process. Lett., vol. 21, no. 12, pp. 1501-1505, Dec. 2014.

[13] Y. Liu, M. Elkashlan, Z. Ding, and G. K. Karagiannidis, "Fairness of user clustering in MIMO non-orthogonal multiple access systems," IEEE Commun. Lett., vol. 20, no. 7, pp. 1465-1468, Jul. 2016.

[14] J. Cui, Z. Ding, and P. Fan, "A novel power allocation scheme under outage constraints in NOMA systems," IEEE Signal Process. Lett., vol. 23, no. 9, pp. 1226-1230, Sep. 2016.

[15] Y. Liu, Z. Qin, M. Elkashlan, Y. Gao, and L. Hanzo, "Enhancing the physical layer security of non-orthogonal multiple access in large-scale networks," IEEE Trans. Wireless Commun., vol. 16, no. 3, pp. 1656-1672, Mar. 2017

[16] Y. Liu, Z. Qin, M. Elkashlan, A. Nallanathan, and J. A. McCann, "Nonorthogonal multiple access in large-scale heterogeneous networks," IEEE J. Sel. Areas Commun., vol. 35, no. 12, pp. 2667-2680, Dec. 2017.

[17] F. Fang, H. Zhang, J. Cheng, and V. C. M. Leung, "Energy-efficient resource allocation for downlink non-orthogonal multiple access network," IEEE Trans. Commun., vol. 64, no. 9, pp. 3722-3732, Sep. 2016.

[18] Y. Zhang, H. M. Wang, T. X. Zheng, and Q. Yang, "Energy-efficient transmission design in non-orthogonal multiple access," IEEE Trans. Veh. Tech., vol. 66, no. 3, pp. 2852-2857, Mar. 2017.

[19] Y. Zeng, R. Zhang, and T. J. Lim, "Wireless communications with unmanned aerial vehicles: opportunities and challenges," IEEE Commun. Mag., vol. 54, no. 5, pp. 36-42, May 2016.

[20] H. Menouar, I. Guvenc, K. Akkaya, A. S. Uluagac, A. Kadri, and A. Tuncer, "UAV-enabled intelligent transportation systems for the smart city: applications and challenges," IEEE Commun. Mag., vol. 55, no. 3, pp. 22-28, Mar. 2017.

[21] M. Mozaffari, A. T. Z. Kasgari, W. Saad, M. Bennis, M. Debbah, "Beyond 5G with UAVs: foundations of a 3D wireless cellular network," May 2018. [Online]. Available: https://arxiv.org/abs/1805.06532

[22] R. Amorim, P. Mogensen, T. Sorensen, I. Z. Kovacs, J. Wigard, "Pathloss measurements and modeling for UAVs connected to cellular networks," in Proc. IEEE Veh. Technol. Conf. (VTC Spring), Sydney, NSW, Australia, Jun. 2017, pp.1-6.

[23] L. Liu, S. Zhang, and R. Zhang, "Multi-beam UAV communication in cellular uplink: cooperative interference cancellation and sum-rate maximization," Aug. 2018. [Online]. Available: https://arxiv.org/abs/1808.00189 
[24] W. Mei, Q. Wu, and R. Zhang, "Cellular-connected UAV: uplink association, power control and interference coordination," to appear in Proc. IEEE Global Commun. Conf. (GLOBECOM), Dec. 2018

[25] J. Lyu, Y. Zeng, R. Zhang, and T. J. Lim, "Placement optimization of UAV-mounted mobile base stations," IEEE Commun. Lett., vol. 21, no. 3, pp. 604-607, Mar. 2017.

[26] A. Al-Hourani, S. Kandeepan, and S. Lardner, "Optimal LAP altitude for maximum coverage," IEEE Wireless Commun. Lett., vol. 3, no. 6, pp. 569-572, Dec. 2014.

[27] R. I. Bor-Yaliniz, A. El-Keyi, and H. Yanikomeroglu, "Efficient 3-D placement of an aerial base station in next generation cellular networks," in Proc. IEEE ICC, Kuala Lumpur, Malaysia, May 2016, pp. 1-5.

[28] Y. Chen, W. Feng, and G. Zheng, "Optimum placement of UAV as relays," IEEE Commun. Lett., vol. 22, no. 2, pp. 248-251, Feb. 2018.

[29] M. Mozaffari, W. Saad, M. Bennis, and M. Debbah, "Unmanned aerial vehicle with underlaid device-to-device communications: performance and tradeoffs," IEEE Trans. Wireless Commun., vol. 15, no. 6, pp. 3949 3963, Jun. 2016.

[30] M. Mozaffari, W. Saad, M. Bennis, and M. Debbah, "Efficient deployment of multiple unmanned aerial vehicles for optimal wireless coverage," IEEE Commun. Lett., vol. 20, no. 8, pp. 1647-1650, Aug. 2016.

[31] K. Li, W. Ni, X. Wang, R. P. Liu, S. S. Kanhere, and S. Jha, "Energyefficient cooperative relaying for unmanned aerial vehicles," IEEE Trans. Mobile Comput., vol. 15, no. 6, pp. 1377-1386, Jun. 2016.

[32] Q. Wu, Y. Zeng, and R. Zhang, "Joint trajectory and communication design for multi-UAV enabled wireless networks," IEEE Trans. Wireless Commun., vol. 17, no. 3, pp. 2109-2121, Mar. 2018.

[33] Q. Wu, and R. Zhang, "Common throughput maximization in UAVenabled OFDMA systems with delay consideration," IEEE Trans. Commun., vol. 66, no. 12, pp. 6614-6627, Dec. 2018.

[34] G. Zhang, Q. Wu, M. Cui, and R. Zhang, "Securing UAV communications via trajectory optimization," in Proc. IEEE Global Commun. Conf. (GLOBECOM), Singapore, Dec. 2017, pp. 1-6.

[35] Y. Zeng and R. Zhang, "Energy-efficient UAV communication with trajectory optimization," IEEE Trans. Wireless Commun., vol. 16, no. 6, pp. 3747-3760, June 2017.

[36] C. Zhan, Y. Zeng, and R. Zhang, "Energy-efficient data collection in UAV enabled wireless sensor network," IEEE Wireless Commun. Lett., vol. 7, no. 3, pp. 328-331, June 2018.

[37] M. A. Abd-Elmagid and H. S. Dhillon, "Average age-of-information minimization in uav-assisted iot networks," Apr. 2018. [Online]. Available: https://arxiv.org/abs/1804.06543

[38] P.K. Sharma, and D. I. Kim, "UAV-enabled downlink wireless system with non-orthogonal multiple access," in Proc. IEEE Global Commun. Conf. Workshops (GC Wkshps), Singapore, Dec. 2017, pp. 1-6.

[39] M. F. Sohail, C. Y. Leow, and S. Won, "Non-orthogonal multiple access for unmanned aerial vehicle assisted communication," IEEE Access, vol. 6, pp. 22716-22727, 2018.

[40] Y. Liu, Z. Qin, Y. Cai, Y. Gao, G. Y. Li and A. Nallanathan, "UAV Communications Based on Non-Orthogonal Multiple Access," IEEE Wireless Commun., vol. 26, no. 1, pp. 52-57, Feb. 2019.

[41] A. L. Yuille and A. Rangarajan, "The concave-convex procedure," Neural Comput., vol. 15, no. 4, pp. 915-936, 2003.

[42] CVX Research, Inc. CVX: Matlab software for disciplined convex programming, version 2.1. Dec. 2018. [Online]. Available: http://cvxr.com/cvx

[43] K.-Y. Wang, A. Man-Cho So, T.-H. Chang, W.-K. Ma, and C.-Y. Chi, "Outage constrained robust transmit optimization for multiuser MISO downlinks: Tractable approximations by conic optimization," IEEE Trans. Signal Process., vol. 62, no. 21, pp. 5690-5705, Nov. 2014.

[44] D. Bertsekas, Nonlinear Programming, 2nd ed. Belmont, MA: Athena Scientific, 1999.

[45] Q. Shi and M. Hong, "Penalty dual decomposition method with application in signal processing," in Proc. IEEE Int. Conf. on Acoustics, Speech and Signal Processing (ICASSP), New Orleans, LA, USA, Mar. 2017, pp. 1-5

[46] Q. Shi, M. Hong, X. Fu, and T. H. Chang, "Penalty dual decomposition method for nonsmooth nonconvex optimization," Dec. 2017. [Online]. Available: https://arxiv.org/abs/1712.04767 